\title{
Wolfgang Christian Schneider \\ Bild und Text in der Silvesterkapelle des päpstlichen Herrschaftsbaus von SS. Quattro Coronati in Rom
}

Nicht weit vom Kolosseum in Rom entfernt, auf einem Sporn des Caelius-Hügels, dem Caeliolus, liegen Kirche und Konvent von SS. Quattro Coronati, ein massiger Gebäudekomplex. Die Anlage geht zurück auf einen Saalbau der Spätantike, der früh mit einer Basilika überbaut wurde. Als Titulus Aemilianae erscheint die Kirche im Liber Pontificalis, sie gehört damit zu den spätantiken Gemeindekirchen in Rom. Schon vor 800 wurde Titulus Aemilianae verdrängt durch den Namen SS. Quattro Coronati. Im Äußeren steht die Anlage heute weitgehend in der Gestalt da, die sie nach der Teilzerstörung des Kirchenbaues von Papst Leo VI. (847-855) in den von Gregor VII. ausgelösten Wirren durch Pascalis II. (1099-1118) erhielt. ${ }^{1}$

Doch nicht der Kirche von Quattro Coronati gelten die folgenden Überlegungen, sondern der Silvesterkapelle in einem an die alte Klosteranlage angelehnten Bau des 13. Jahrhunderts. Eine heute im Inneren erhaltene Inschrift gibt an, ${ }^{2}$ dass dieser hochragende Gebäudeverband an dem alten Benediktinerkonvent, Turmburg mitsamt der Kapelle („,capellam et domos“) und einem zugehörigen darüberliegenden Saal, um die Mitte des 13. Jahrhunderts eigens - vom Kloster rechtlich unabhängig - angelegt worden ist, um nach Innozenz' IV. Abzug von Rom anstelle des nicht zu verteidigenden Lateranpalastes als ,Zitadelle“ für den von Innozenz als Stellvertreter in der Stadt zurückgelassenen Kardinalpresbyter Stephanus von S. Maria in Trastevere aus der Familie der Conti zu dienen. Nicht um irgendeine abgelegene Kapelle also geht es, sondern um einen Herrschaftsraum, und zwar den einzigen päpstlichen Herrschaftsraum des Hochmittelalters, der erhalten blieb: der Ort, der den Papstsitz im Lateran im Falle einer existentiellen Bedrohung der päpstlichen Herrschaft zu vertreten hatte und daher auch rechtlich dem Lateran, der Kirche der römischen Bischöfe zugehörte. ${ }^{3}$

Wer heute in die Silvesterkapelle eintritt, einen einschiffigen tonnengewölbten Raum von $9,15 \times 4,80 \mathrm{~m}$, sieht sich von einem leuchtenden Fries von Fresken umgeben, der den ganzen Raum durchzieht. ${ }^{4}$ Sie sind berühmt, wenngleich letztlich

1 Grundlegend zum Bau: Muñoz 1914; Ghetti 1964; Manzi 1968; Krautheimer 1970, 1-36. Zu den Neufunden über der Kapelle: Draghi 2006. - Diese Darlegungen gründen auf Gastvorträgen zum politischen Gehalt der Büsten-Medaillons, die ich 2004 an der Universität Frankfurt (Johannes Fried) und im Roemer- und Pelizäus-Museum Hildesheim, sowie 2006 an der Universität Karlsuhe (Norbert Schneider) gehalten habe.

2 Zu der in die Südwand eingelassenen Bauinschriften: Forcella 1876, 290 Nr. 718; 719.

3 Dies übersieht Noll 2011, 125-127.

4 Zur Malerei vgl. Muñoz 1913; Matthiae 1964, 86-91. Matthiae 1966, 146-152. Mitchell 1980 (mit 23 Abb.); Sohn 1997; Noll 2011.

Ә Open Access. (c) 2019 Wolfgang Christian Schneider, publiziert von De Gruyter. (c) BY-NC-ND Dieses Werk ist lizenziert unter der Creative Commons Attribution-NonCommercial-NoDerivatives 4.0 Lizenz. 
nur oberflächlich in ihrem Gehalt erörtert. Erst dem genaueren Blick wird auffallen, dass unter den Vollbildern eine Reihe von Tondi mit Büsten entlangläuft, die meisten sind allerdings heute weitgehend zerstört. Jeder dieser Tondi enthält eine Büste, die ein Schriftband vor der Brust ausbreitet. Es steht also ein hochherrschaftlicher sakraler Schriftraum vor dem Betrachter. Ihn gilt es zu erläutern, und zwar im Hinblick auf die Interdependenz zwischen den Vollbildern und den Büsten mit ihren Schriftbändern im Raum. Denn die so entstandene Text-Bild-Anlage ist in beispielhafter Weise geeignet, die Bedeutung räumlich organisierter Schriftlichkeit aufzuweisen - und zwar gerade auch als Mittel im politischen Ringen zwischen Kaisertum und Papsttum. Die Schriftteile stehen dabei in einer Spannung zu den Bildteilen, die sie schärfen und die in ihrer Aussage daher zunächst zu betrachten sind.

Die szenischen Fresken bieten an der Westwand, durch die man eintritt, Geschehnisse der Actus Silvestri, der Legende von Silvester, dem römischen Bischof, unter dem die Kirche in die römische Staatlichkeit eintritt. Die Legende über die wunderbare Bekehrung Kaiser Konstantins durch Silvester wurde in der Spätantike aus einzelnen älteren Überlieferungen zusammengestellt. In karolingischer Zeit gingen Teile der Silvesterlegende dann in das sogenannte Constitutum Constantini ein: die „Konstantinische Fälschung“, die von Männern der kirchlichen Opposition gegen Ludwig den Frommen im 9. Jahrhundert hergestellt wurde. ${ }^{5}$ Nachdem die Konstantinische Fälschung in der Ottonenzeit in kirchenpolitischen Zusammenhängen gelegentlich Erwähnung fand, wurde sie seit dem 11. Jahrhundert von kurialer Seite begeistert aufgegriffen und - tendenzhaft umgearbeitet - beharrlich aufgerufen, um die Vormachtstellung des römischen Bischofs und dessen Überordnung über den Imperator zu belegen: Hier erst setzt die eigentliche „Fälschung“ ein. Eine erste bildliche Ausformung samt einer Inschrift scheint die Fälschung in der Zeit Kaiser Lothars III. im Lateran, erfahren zu haben, die auf Vorgänge zwischen Lothar und Innozenz II. in Lüttich 1131 bzw. auf Lothars Romaufenthalt 1133 Bezug nahm, doch wurde dieses Werk bzw. die dieses ausdeutende Inschrift als anmaßend und die rechtliche Situation verfälschend wohl zwischen 1154 und 1157 von kaiserlichen Truppen zerstört. ${ }^{6}$ Die im 13. Jahrhundert geschaffenen Fresken der Silvesterkapelle müssen also als eine Neuauflage dieser untergegangenen propagandistischen Bildwerke aufgefasst werden. Für sie sind, wie sich zeigen wird, beide eben genannten Texte wirksam geworden und dementsprechend für die Deutung heranzuziehen.

5 Fried 2007, bes. 112-114; Fried 2011, S. 295-311.

6 Walter 1970, 168f. Siehe auch Schramm 1983, 256 und Abb. 198. 


\section{Die szenischen Bilder der West- und Nordwand: Die Legende von Konstantin und Silvester}

Der Zyklus der Vollbilder setzt in der Ecke des Südteils der Westwand ein, um dann zur Nordwand hin voranzuschreiten (Abb.1). Im ersten Bild sitzt der von Gott wegen der sündhaften Verfolgung der Christen mit dem Ausschlag gezeichnete Kaiser Konstantin auf seinem Thron; neben ihm klagen Mütter mit ihren Kindern, da der Kaiser, dem von heidnischen Priestern gesagt wurde, er könne durch ein Bad in Kindesblut genesen, ${ }^{7}$ dreitausend Kinder hatte zusammenführen lassen. Bewegt vom Jammer der Mütter lässt Konstantin jedoch von seinem Vorhaben ab und entlässt die Flehenden.

Die anschließende Szene zeigt den Wendepunkt an: Dem schlafenden Kaiser erscheinen - gleichsam von dem darüber dargestellten im Weltgericht thronenden Christus herabgesandt ${ }^{8}$ - Petrus und Paulus und verkünden ihm die Heilung durch

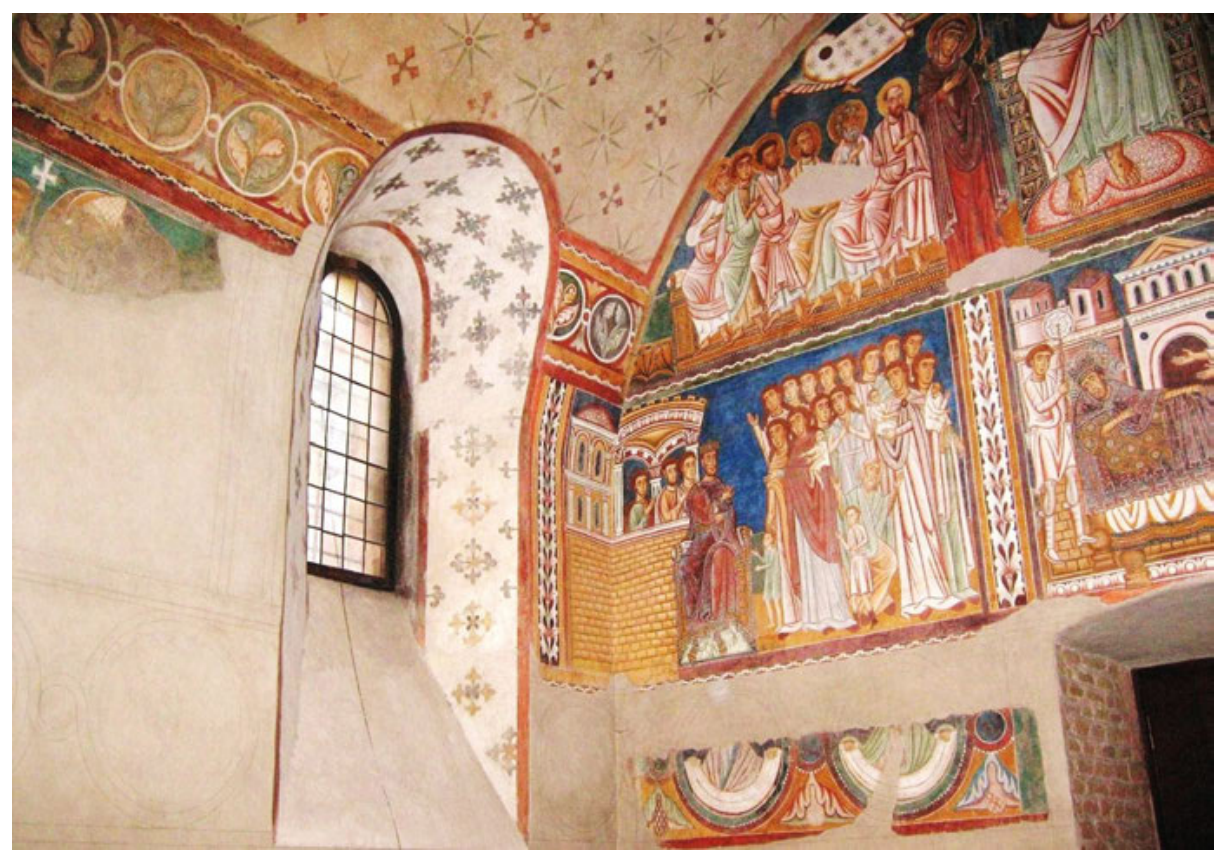

Abb. 1: Silvesterkapelle, Südwestecke: Konstantin und die klagenden Mütter, darunter die Reste der Tondi (v. l.) von ISAAC und ABRAAM.

7 Die Kunde der heidnischen Priester ,verballhornt ' das christliche Taufgeheimnis: das reinigende Bad im Born des durch das blutige Selbstopfer des Menschensohnes erlangten Taufwassers.

8 Von der Herabsendung durch Christus spricht die Legende wie auch der Constitutum-Text, vgl. Fuhrmann, Constitutum, $\S 7$. 


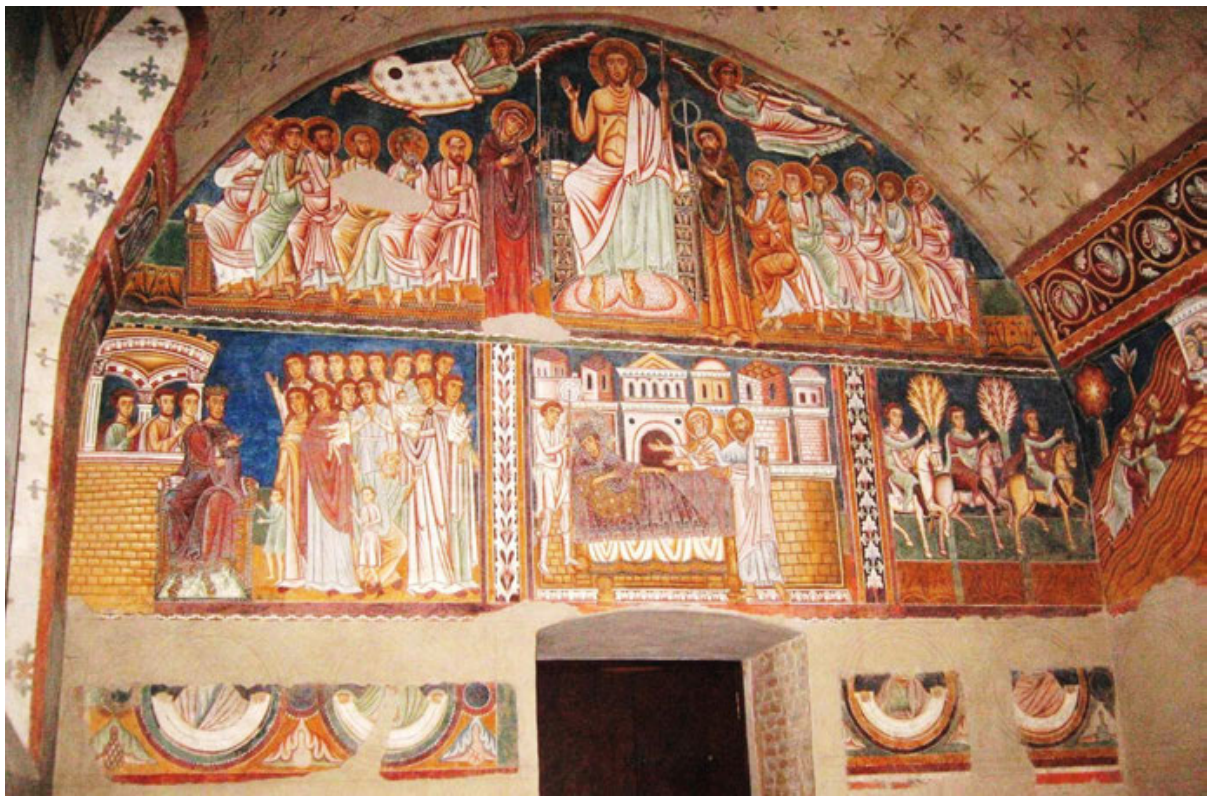

Abb. 2: Silvesterkapelle, Westwand: Oben Weltgericht Christi, darunter Konstantins Traum. darunter die Reste der Tondi (v. I.) von ISAAC und ABRAAM, dann DAVID und SALOMON.

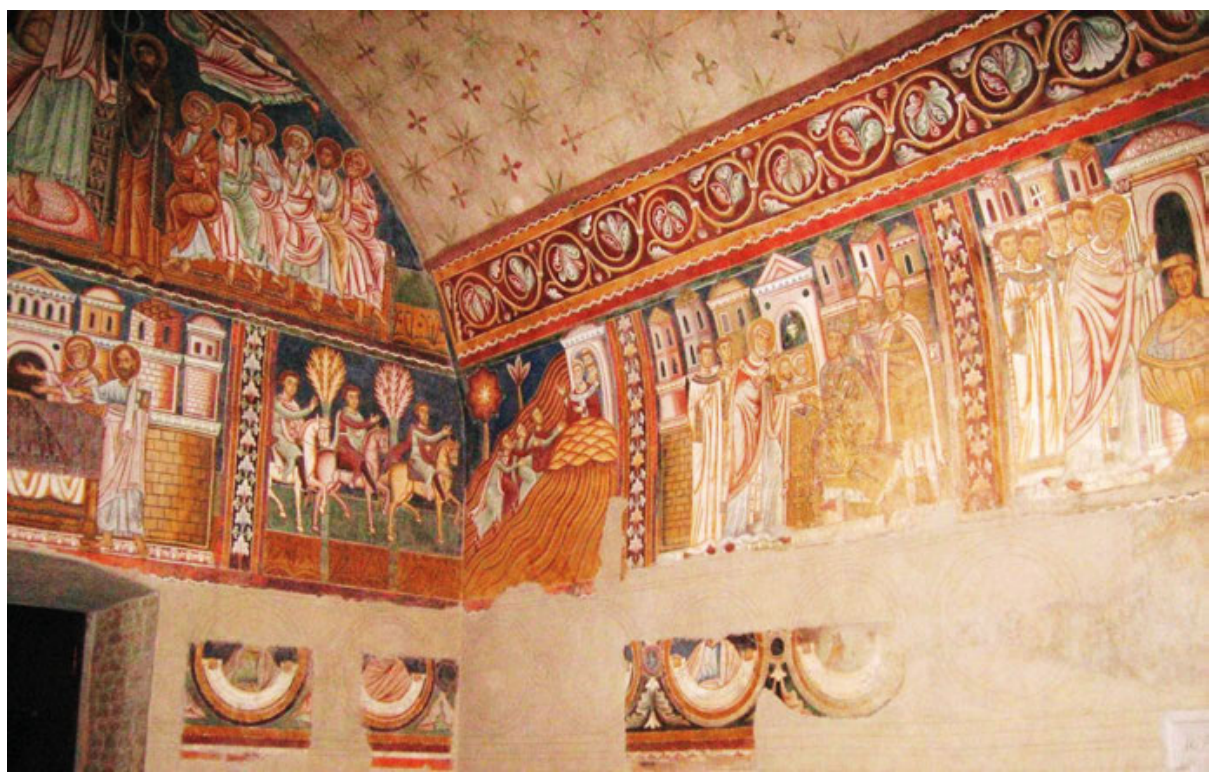

Abb. 3: Silvesterkapelle, Westnordecke: Die Boten an Silvester, Vorweisung der Bildnisse von Petrus und Paulus; darunter die Reste der Tondi (v. I.) von DAVID und SALOMON, sowie nach der Lücke IEREMIAS und EZECHIEL. 


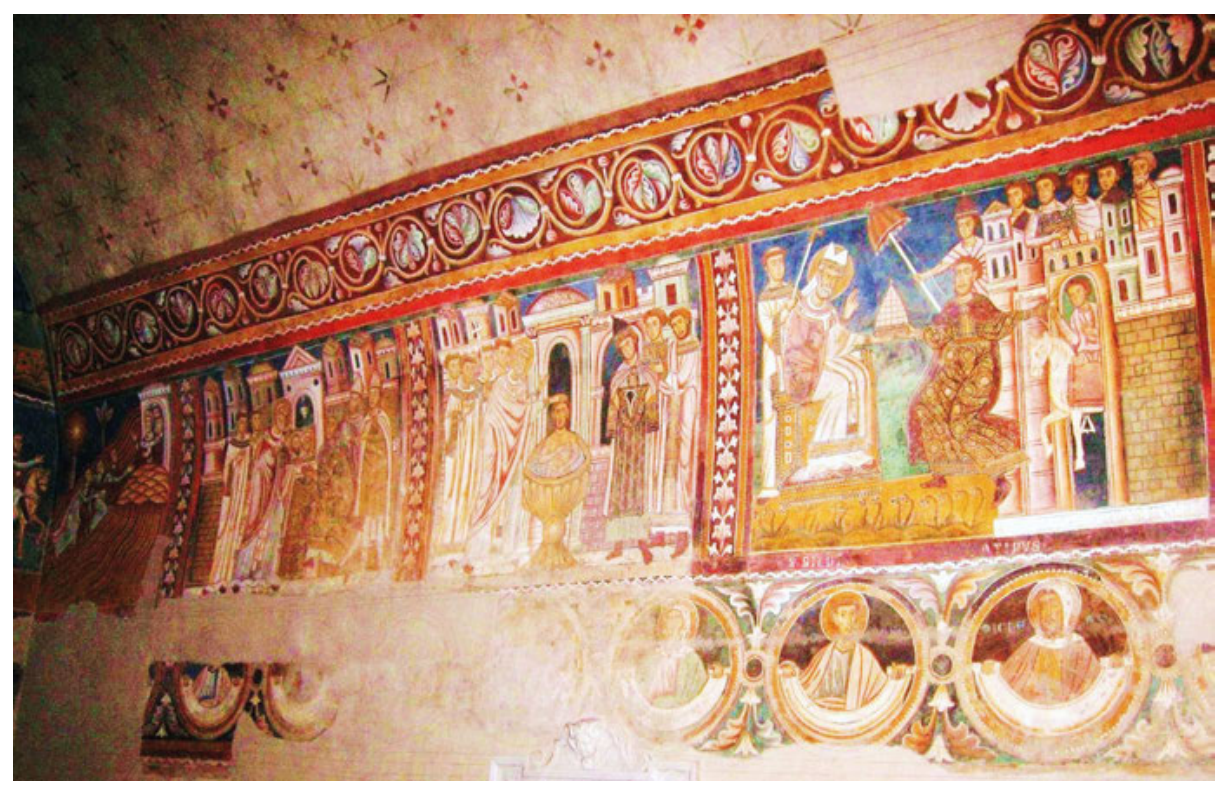

Abb. 4: Silvesterkapelle, Nordwand: Vorweisung der Bildnisse von Petrus und Paulus, Taufe Konstantins; unter letzteren die Tondi von AMOS, IONAS, MICHEAS.

das Bad in einem Brunnen der pietas, ${ }^{9}$ sobald er den wegen der kaiserlichen Verfolgungen auf dem Soracte verborgenen Silvester, den Bischof von Rom, zu sich berufen hätte (Abb.2).

Das nächste, zur Nordwand umknickende Doppelbild zeigt die Reise der drei vom Kaiser ausgesandten Boten zum Soracte und ihre demütige Bitte vor Silvester, seinen Zufluchtort zu verlassen und dem Kaiser zur Heilung zu verhelfen (Abb.3). Dieser Vorgang wird allein in der Silvesterlegende geschildert.

Nach seiner Rückkehr nach Rom weist Silvester auf dem anschließenden Fresko der Nordwand dem Kaiser auf dessen Bitten die Bilder der beiden Apostel; ergriffen erkennt der gekrönte, aber noch immer aussätzige Konstantin in ihnen die Verkünder seiner nahen Heilung (Abb.4). Zwei Geistliche und zwei Höflinge (mit der spitzen Kopfbedeckung von duces) sind Zeugen des Geschehens innerhalb eines Kirchenraums, wohl der Laterankirche: Deutlich sind hinter dem Doppelbild der Apostel (oben) das Ziborium zu erkennen und (unten) die Türen der Altarschranke.

Die folgende Szene zeigt den Kaiser bloßen Leibes im ,Bad der Pietas', vom Aussatz nun befreit, auf seinem Haupt liegt die Hand Silvesters, der eben den Taufakt vollzieht. Hinter dem Bischof begleiten vier Geistliche den Akt, ihnen gegenüber stehen drei hochrangige Höflinge mit den Insignien des Kaisers: Der Träger einer

9 Fuhrmann, Constitutum, § 7: fons pietatis. 


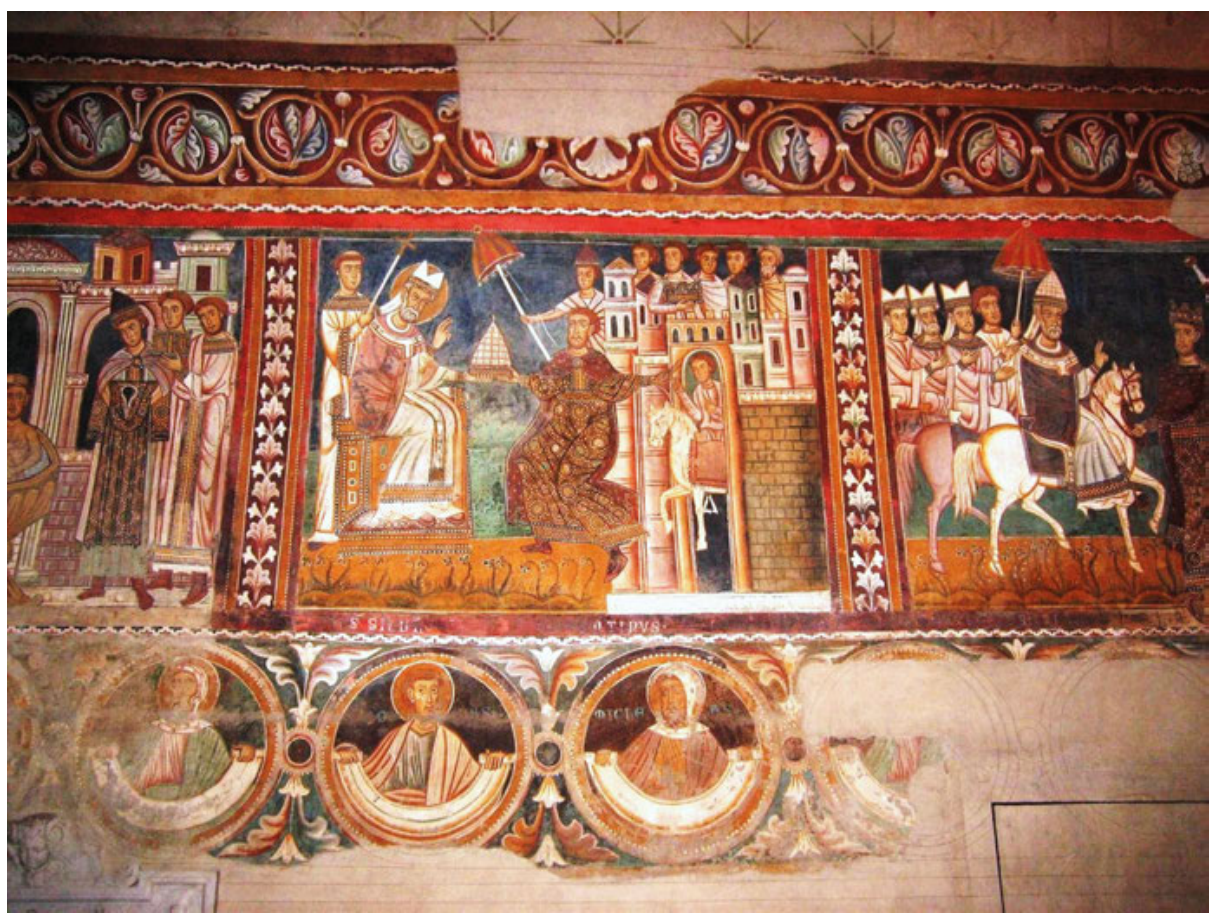

Abb.5: Silvesterkapelle, Nordwand: Die Tiaraübergabe an Silvester; darunter die Tondi von IONAS, MICHEAS.

purpurnen Tiara hält seine Dalmatica, ${ }^{10}$ ein zweiter mit breitem Schmuckstreifen am Gewand die Krone bereit. Die Kuppel hinter dem Täufling scheint einen Baptisteriumsbau (vielleicht erneut den des Lateran) anzeigen zu wollen.

Die auf die Taufe folgenden Bilder, die beiden letzten Bildszenen auf der Nordwand (Abb.5), folgen allein der Constitutum-Fälschung, die zu einem Teil spätantike oströmisch-byzantinische Erzählungen über den ,Umzug' des Kaisers in den Osten einbezieht. Denn obwohl die Silvesterlegende ausführlicher auf Geschehnisse nach der Taufe eingeht, sagt sie nichts, was in Richtung des Inhalts der beiden nächsten Bilder weisen könnte; mehr noch: Da sie zahlreiche kirchenfreundliche Akte des Kaisers beschreibt, etwa Besitzübertragungen und das Versprechen von Kirchenbauten, ist letztlich auch kein Raum für polemische Inhalte.

Das erste dieser beiden Bilder ist das bekannteste des Freskenzyklus: Silvester sitzt auf einem Thron, der dem des Kaisers auf dem ersten Bild des Zyklus ähnelt. Der Kaiser schreitet aus einer befestigten Toranlage heraus auf ihn zu und übergibt ihm, in einer Haltung, die ansonsten - vor allem in den Buchübergaben in Widmungsszenen - gebende, mitunter auch nachgeordnete Personen einnehmen, besondere

10 Man könnte in ihm den später belegten Patricius der Römer erkennen. 


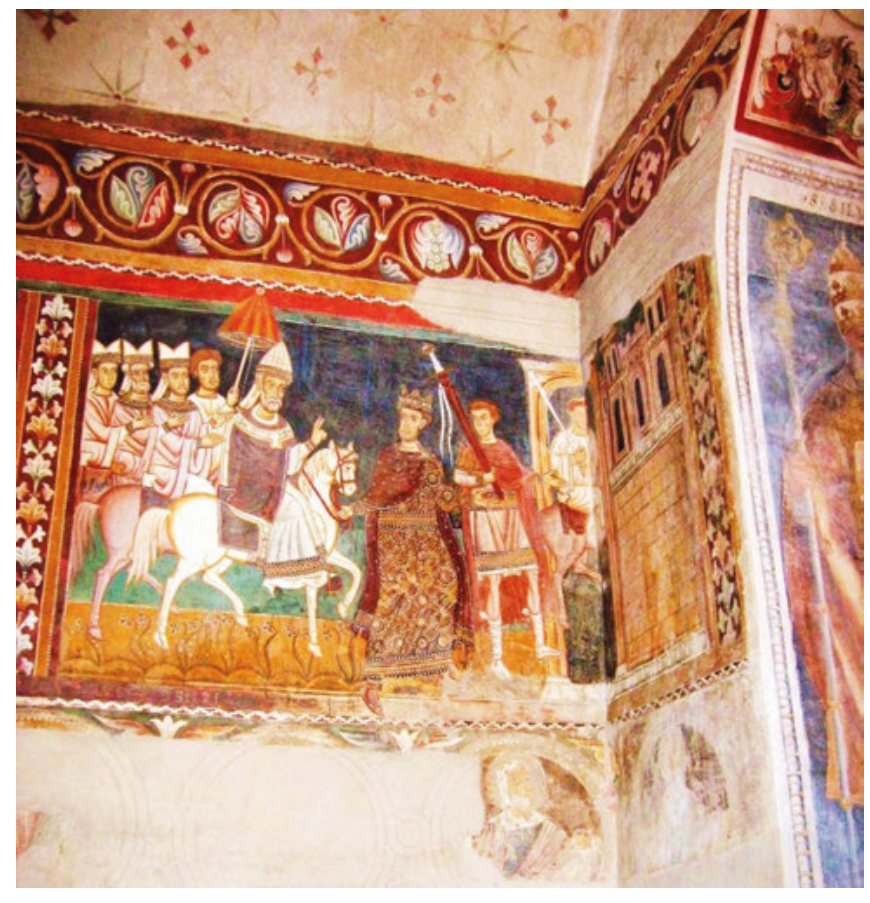

Abb. 6: Silvesterkapelle, Nordostecke: Der StratorDienst Konstantins; darunter die Tondi von SOPHONIAS und (um die Ecke) AGGEUS.

Ehrenzeichen, offenkundig als Dank für die erlangte Heilung: das phrygium, eine ausgezierte Tiara, mit der Rechten, ein eben aus dem Tor tretendes weißes Ross mit der Linken und wohl auch den von einem Mann des kaiserlichen Gefolges dem Thronenden entgegengehaltenen Ehrenschirm, der auf dem folgenden Fresco im Gefolge Silvesters erschient. Hinter dem Kaiser erhebt sich die Stadt, ein vereinzeltes $C$ im Schriftband (unter dem Tor) könnte auf „Capitolium“ (oder „Palacium“) hinweisen), ${ }^{11}$ von den Zinnen sieht die Bevölkerung oder die Getreuen des Kaisers dem Geschehen $\mathrm{zu}$, einer von ihnen hat die Krone des Kaisers in der Hand, was kaum anders zu verstehen ist, als dass diese Krone in den gezeigten Akt nicht einbezogen ist, also unverrückt in der Verfügung des Kaisers liegt. ${ }^{12}$ Der dargestellte Übergabevorgang selbst und die drei hier im Bild auftretenden Rangeszeichen haben zunächst nichts übermäßig Auszeichnendes und den Empfänger Überordnendes an sich. Das spätantike Imperium kannte Ehrengaben und ehrende Erlaubnisse, so war auch der Gebrauch

\footnotetext{
11 Es handelt sich wohl um das C, das Kantorowicz 1961, 181 als Beginn des Wortes „Capitolium“ deutete, das Sohn 1997, 31, Anm 109 vermisst. Erhalten sind noch Teile der Benennungen: S. Silvester und Constantinus.

12 Zu den Kaiser-Papst-Bildern aus kurialer Perspektive: Sohn 1997, 31-39, wiederholt unter Rückgriff auf Texte Innozenz' III. Noll 2011, 44-46 will die dargestellten Schenkungen auch bildlich recht weit ausgedehnt ansehen, was nicht überzeugt. Er denkt zu sehr vom Text des Constitutum her, bei dem die Sachlage eine andere ist. Das Bild in der Kapelle ist zurückhaltender.
} 
von Pferden beschränkt, nur Höherrangigen war ihre Nutzung gestattet. ${ }^{13}$ Nachdem die christlichen Bischöfe - im Zuge des Eintritts der Kirche in die Staatlichkeit - ranglich auf die Ebene der Provinzstatthalter gehoben wurden, kam ihnen, also auch dem römischen Bischof, ganz folgerichtig von Seiten des Kaisers ein Pferd zu.

Das ähnlich berühmte letzte Bild der Nordwand schließlich zeigt Kaiser Konstantin, der gekrönt und in Begleitung eines Schwertträgers das Ross, auf dem Silvester sitzt, zu Fuß am Zügel zur Stadt hinführt (Abb. 6). Ein Diakon mit einem Kreuzstab reitet voran. Hinter Silvester folgen Männer bischöflichen Ranges, wohl die im gefälschten Constitutum ebenfalls bedachten, den Senatoren und Consuln ranglich gleichgestellten ,Kardinäle‘. ${ }^{14}$

Diese Szene, die in der Silvesterlegende nicht nur fehlt, sondern dort nicht einmal einen Ansatzpunkt besitzt, geht allein auf das gefälschte Constitutum Constantini zurück, wo erwähnt wird, Constantin habe zugleich mit der Übergabe des phrygiums aus Verehrung für den Beatus Petrus die Zügel von Silvesters Pferd gehalten und dem Bischof das officium stratoris geleistet. ${ }^{15}$

Allerdings ist das Motiv des Geleitens hinsichtlich der Stellung der Beteiligten keineswegs so eindeutig, wie es die spätere mittelalterliche Deutung der Kirche wollte. Oft genug ist der fürsorgende Führer der eigentlich Übergeordnete, der anscheinend herrscherlich Geführte aber der eigentlich Untergeordnete. Das zeigt deutlich die Motivgeschichte, die Kantorovicz in einem Aufsatz zur Strator-Szene erläuterte. ${ }^{16}$ Er zeigte, dass die Ross-Führung in der Antike durch übergeordnete Personen erfolgt: auf Gedenkprägungen etwa führt die Göttin Victoria das Ross des Imperators, häufiger auch treten die Dioskuren als Rossführer auf. Auch aus diesem Grunde überrascht nicht, dass - wie Holtzmann feststellte ${ }^{17}$ - das officium stratoris ${ }^{18}$ für die päpstliche Seite an Bedeutung verlor und sie lieber das officium strepae gesehen hätte, den Dienst der Steigbügelhaltung, der wirklich ein entschieden rangminderndes Tun war. Aber davon sprach nicht einmal das Constitutum, konnte es auch nicht, weil zu der Zeit, als die Fälschung gearbeitet wurde, die Steigbügel weitgehend unbekannt waren, und das 4.Jahrhundert, das für die päpstliche Fälschung ja gelten sollte, vom

13 Vgl. Mommsen, Codex Theodosianus VIIII 30, 1-5.

14 Fuhrmann, Constitutum, § 15.

15 Fuhrmann, Constitutum, § 16, Z. $257 \mathrm{f}$.

16 Kantorowicz 1961, 181-189.

17 Es geht dabei, wie Mitchell 1980, 26 mit Anm. 42 feststellt, um eine Verrechtlichung älterer, relativ offener personaler Akte in einer feudalen Ordnung, insofern versuchte die Kurie tatsächlich die Rechtsordnung verfälschend zu verschieben; zu unklar ist dies bei Holtzmann 1928; angemessener bei Eichmann 1930, 16-40. Holtzmann 1932.

18 Sohn 1997, 38 und Noll 2011, 47 übersetzen strator hochproblematisch mit „Reitknecht“, richtig aber ist „Wegbereiter“, was einen ganz anderen Horizont eröffnet, eben den von Kantorowicz behandelten. Neben Konstantin steht kein „Offizier“, sondern der (dukale) Schwertträger des Kaisers. Dass das Schwert auf den Papst zu beziehen ist, so Noll 2011, 49, ist abwegig. 
Steigbügel nicht das geringste wusste. Man muss davon ausgehen, dass die Kaiser des hohen Mittelalters diesen Akt nie vollzogen.

\section{Die szenischen Bilder der Südwand: Die Helena- und Kreuzlegende}

Nach den beiden allein dem Text der Constitutum-Fälschung folgenden Szenen kehrt die Bildfolge der Silvesterkapelle auf der Südwand wieder zur Silvesterlegende zurück. Alle drei Szenen der Südseite, die kaum je eingehender erörtert werden, fallen in die Zeit nach den Ereignissen der Nordseite. Die Bildfolge setzt ein mit dem Stierwunder der Silvesterlegende (Abb. 7). ${ }^{19}$

Kaiserin Helena hatte sich dem Judentum angeschlossen und wegen Konstantins Götterverehrung den Kaiserhof verlassen. Von ihrem Sohn von seinem Glaubenswech-

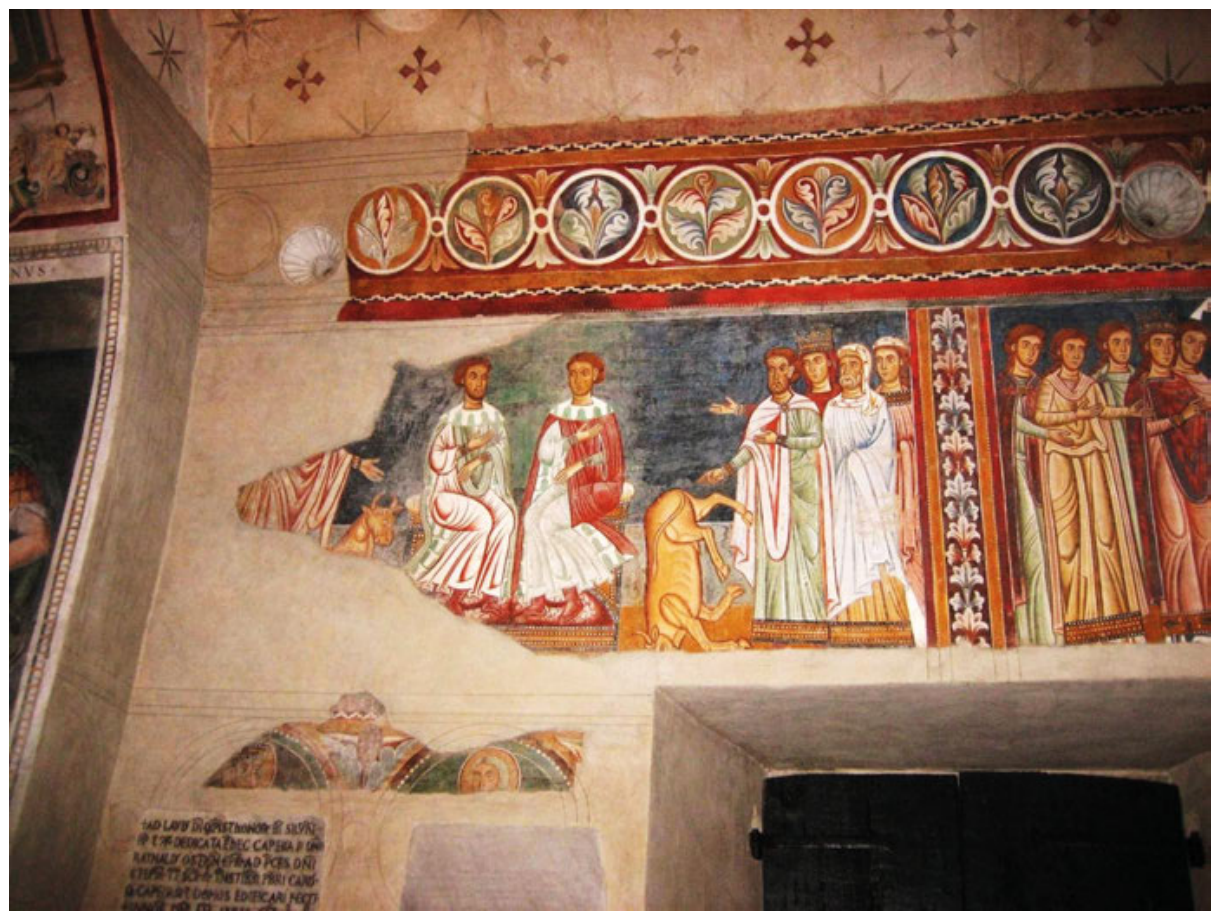

Abb. 7: Silvesterkapelle, Südwand: die Disputation zwischen Silvester und Zambri; darunter die Tondi von JOSIAS und EZECHIAS.

19 Die (wiederholt überarbeitete) Silvester-Vita zugänglich in Mombritius 1910, 508-531, hier 515ff. 
sel unterrichtet, lobte sie zwar dessen Bekehrung zur Verehrung des Einen Gottes, erklärte die Entscheidung für das Christentum jedoch für die falsche Wahl. Daraufhin wurde eine Disputation zwischen den jüdischen Priestern unter Führung Zambris aus Helenas Hofstaat und Silvester von Seiten des Sohnes in Rom vor den von Konstantin eingesetzten heidnischen Richtern Craton und Cenophiles verabredet. Diese Personen zeigt das Fresko; von Silvester ist heute noch die linke Hand mit dem Mantelansatz und der Kopf des Stiers zu sehen, auf der - im Folgenden näher behandelten - Kopie des Freskos von Antonio Eclissi von 1637 sind allerdings noch fast die gesamte Gestalt des Papstes und auch sein geistlicher Anhang sowie die Hälfte des Stiers überliefert. ${ }^{20}$ Als aber Zambri Silvester in der Disputatio nicht standzuhalten vermochte, suchte er durch die Vollbringung eines Zeichens die Macht seines Glaubens zu erweisen: er flüsterte einem Stier ein Wort in das Ohr, worauf das Tier tot zu Boden fiel. Das Fresko zeigt den Stier kopfüber gestürzt. Doch der jüdische Priester vermochte nicht, wie Silvester - um die göttliche ,Herkunft‘ des tötenden Wortes zu klären - forderte, den Stier nun wieder zum Leben zu erwecken. Das aber gelang Silvester mit seinem Wort, auf dem Fresco erscheint daher unter der geöffneten Hand Silvesters der Kopf des wiedererweckten Stieres. Damit hatte Silvester Macht und Richtigkeit der christlichen Gottesverehrung erwiesen, der sich nun auch Helena und ihr (hinter Zambri auftretender) Hofstaat anschlossen.

Die anschließende Szene (Abb. 8), die auch unter dem Bild noch in Resten benannte ,Kreuzauffindung‘, fehlt im Constitutum, sie entstammt einer dritten Quelle: der Helena-Legende. Diese ist zwar zunächst vor allem mit der Kirche S. Croce in Gerusalemme verbunden, hat aber auch für die Silvesterkapelle eine erhebliche Bedeutung, besitzt die Kapelle doch, wie die schon erwähnte Widmungsinschrift in der Kappelle selbst berichtet, nicht nur Silvester-Reliquien, sondern vor allem auch - nach der benachbarten Inschrift - eine Reliquie des Hl. Kreuzes (de ligno crucis).

Die zum Christentum bekehrte Helena war nach Palästina gezogen, um dort in Jerusalem das Kreuz Jesu zu suchen. Eben wird in Gegenwart der Kaiserin und ihrer vier Begleiterinnen auf der einen, drei Männern des Hofstaates auf der anderen Seite das dritte Kreuz ergraben, das nun, wie seine hohe Position sowie die Geste der Kaiserin zeigen das Kreuz Jesu ist: es hat dieselbe Gestalt wie alle bisher im Aufzug Silvesters auftretenden Vortragekreuze. An den Erdhügel gelehnt wartet der Lahme mit deutlich leblos-weiß gegebenen Beinen, der durch seine wunderbare Heilung die Echtheit des geborgenen Kreuzes erweisen wird. Das Auftreten dieser Szene im Rahmen der Silvesterlegende - zusätzlich dadurch hervorgehoben, dass die Kreuzauffindung der wichtigsten politischen Szene der Nordwand gegenüberliegt - setzt einen besonderen, die engere Silvestergeschichte überschreitenden Akzent und zeigt

20 Eclissi fol.24. Die Richter waren nach Ausweis der Nachzeichnungen im Band unter der Szene als solche inschriftlich benannt. Abgesehen davon ist von den Wörtern des unten durchlaufenden Schriftbandes auf der Südseite nur noch der Rest von taurus (trs) und inventio sanctae crucis unter dem Folgebild erhalten. 


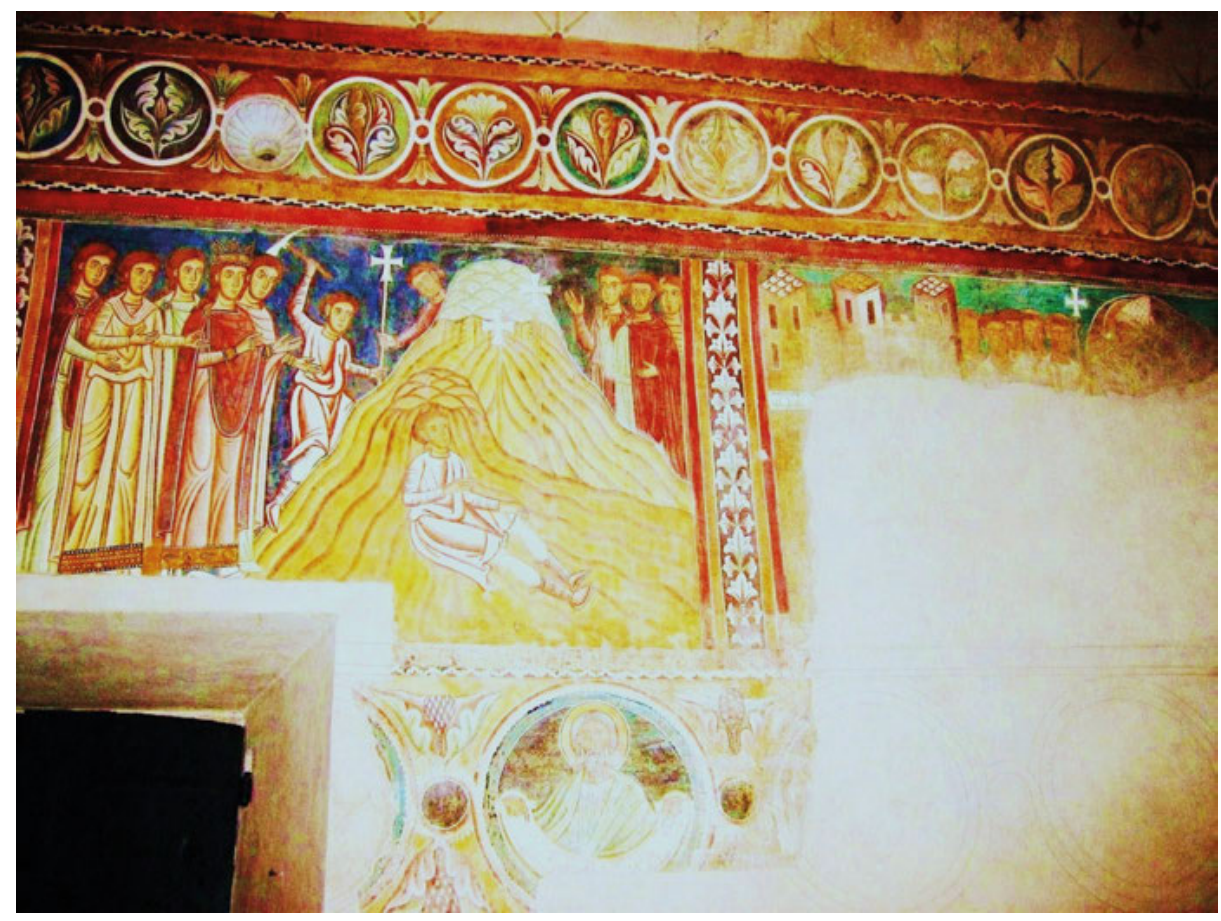

Abb. 8: Silvesterkapelle, Südwand: Kreuzauffindung durch Helena und Fesselung des Drachens; darunter der Tondo von SAMUEL.

damit an, dass die Darstellungsabsicht der Fresken über die schlichte Illustration der Silvester-Geschehnisse hinausgeht.

Das letzte Bild der Südseite ist heute großteils verschwunden. Schon vor dem 15. Jahrhundert wurde es an seinem rechten Rand für eine Vergrößerung des Fensters beschnitten, Weiteres ist zerstört. Doch das Verbliebene gibt genügend Hinweise, um den Inhalt des Dargestellten verstehen zu können. Die erhaltene obere Zone des Fresco zeigt links einen Mauerring mit Türmen, von dem ein Zug von sechs Geistlichen ausgeht. Er wird angeführt von einem Tiaraträger, also Silvester selbst, dessen Oberkörper seitlich vor einer nahezu halbrunden dunklen Fläche steht. Dem Bischof folgt ein tonsurierter geistlicher Kreuzträger, neben dem ein weiterer Pallienträger steht. Von den drei Personen in der zweiten Reihe wird noch ein weiterer durch die Tonsur als Geistlicher ausgewiesen. Rechts oben waren - Eclissis Kopie zufolge, doch heute verloren - mehrere Baumwipfel zu sehen. Den entscheidenden Hinweis für die Deutung des Bildes gibt die hinter dem Oberkörper Silvesters gezeigte annähernd halbrunde dunkle Fläche. Denn die Silvesterlegende berichtet, ${ }^{21}$ dass der römische Bischof unter dem Beistand Petri geleitet von zwei Diakonen und drei Priestern zu

21 Pohlkamp 1983. 
einem das Volk von Rom bedrohenden Drachen hinabstieg und diesen mit Fesseln band - zweifellos mit Hilfe des Kreuzes. Die Legende berichtet ja, dass das Kreuz Christ auf dem Siegelring stand, der die Fesselung des Drachens siegelte. Die deutlich wiedergegebene dunkel getönte Fläche hinter Silvester dürfte die Höhle dieses Drachens anzeigen, der sicherlich im zerstörten Bereich als gebunden dargestellt wurde.

Mit seinem Sieg über den Drachen und dessen Fesselung und Einschließung bis zum Gericht, ist das Vermögen Silvesters erwiesen, mit der ihm eigenen Macht und der Verfügung über das Kreuz jedes menschenbedrohende Widerwesen in Bann zu schlagen. Im Hinblick auf das voraufgehende Bild der Kreuzauffindung ist das von Silvester in der Drachenszene mitgeführte Kreuz also ,scharf‘ zu deuten: als das Kreuz der Kreuzreliquie, die Helena nach Rom übertragen hatte, womit als vierte Vorlage die römische Lokaltradition aufgegriffen worden wäre, die sich um die Kirche S.Croce in Gerusalemme rankt und im Liber Pontificalis ihren Niederschlag fand. Dies allein kann die Aufnahme der innerhalb der Silvesterlegende nachrangigen und spät auftretenden Episode der Kreuzauffindung in den Zyklus erklären: Mit der Aufnahme dieser in der Folge der Fresken eigentlich irritierenden Episode haben Planer und Maler der Darstellung versucht, den römischen Bischof und sein Tun nicht nur mit dem höchsten Herrscheramt - mit dem Kaisertum - in Verbindung zu bringen, sondern ihn auch auf engst mögliche Weise mit dem Kreuz Christi, dem vornehmsten und ranghöchsten Gegenstand des Heilsgeschehens, zu verbinden und dieses als in der Hand des Papstes, in seiner Verfügung stehend, zu bestimmen.

\section{Das Herrschaftsbild Christi im Giebelhalbrund der Westwand}

Ihren Endpunkt und ihr Ziel haben die Bildszenen dann im Halbrund oben in der Westwand im endzeitlichen Herrschaftsbild Christi: Sie motivieren das auf den Westseiten mittelalterlicher Kirchen traditionelle ,Weltgerichtsbild“ auf ganz spezifische Weise - und das erklärt seine Besonderheiten. Nicht einfach ein Weltgerichtsgeschehen ist gemeint, wie es - annähernd zur selben Zeit wie in der Silvesterkapelle - im Dom von Torcello auftritt, ${ }^{22}$ dargestellt ist vielmehr die Vergegenwärtigung von Christi Allmacht nach seinem Selbstopfer, im herrscherlichen Thronen mit den Insignien seines Heilshandelns, den Arma Christi am Thron - eine ikonographisch einmalige Darstellung -, deren Vornehmstes, das Kreuz, in der Hand Christi steht, das zugleich im Bildfries darunter als konkret gefunden und in der Verfügung des Papstes stehend gezeigt wird. Die Gottesgebärerin und der Vorläufer umgeben den Herrn stehend im Sinne einer Deesis bei Weltgerichtsbildern, ihnen folgen auf der Thronbank die

22 Zu Torcello: Concina 1996, 112-123. 
Apostel, über denen die Engel der Apokalypse schweben. Doch nicht eigentlich ein richterliches Tun ist gezeigt, trotz der Engel, die endzeitlich die Posaune blasen und den Himmel aufrollen. Denn Gesegnete oder Verdammte treten nicht auf. Es geht um ein herrscherliches Walten in der Endzeit und Ewigkeit.

Die über die Wände verteilten aus vier Silvester-Texten gezogenen Vollbilder erweisen somit die ausgemalte Kapelle als einen semantisch geschlossenen Gesamtraum, in dem das Einzelbild nicht unabhängig vom Gesamtzusammenhang der Ausmalung betrachtet werden kann. Inhalt der Bildfolge ist offensichtlich die Stellung Silvesters, und zwar wesentlich im Hinblick auf seine Zugehörigkeit zu den Aposteln Petrus und Paulus sowie seine Verfügung über das Kreuz Jesu. Dem Besucher von heute fällt allerdings kaum auf, dass unter der Reihe dieser szenischen Bilder eine Folge von Büstenmedaillons (bzw. Tondi) entlangzieht, ${ }^{23}$ die dem offenkundig geschlossenen Bildzusammenhangs gleicherweise konstitutiv zugehören müssen.

\section{Die Folge der Büstenmedaillons}

Wenig ist heute von diesen ursprünglich mindestens 30 Tondi erhalten und so fanden sie bis vor kurzem bei der Würdigung und Deutung der Fresken in der Silvesterkapelle keine Berücksichtigung. ${ }^{24}$ Und doch sind sie für das Verständnis der Silvesterkapelle von entscheidender Bedeutung: Denn der so gegebene Gestalt-Zusammenhang von szenischer Bildzone und darunter liegender Tondi-Reihe ist insgesamt in der frühen Kunst nicht sehr häufig, doch er begegnet gerade in Rom, sowohl in der römischen Bischofskirche des Lateran und in der Grabeskirche des Apostels Petrus, wie frühneuzeitliche Zeichnungen überliefern, als auch in St. Paul vor den Mauern und in S. Croce in Gerusalemme. Wenngleich im Maßstab verringert, stellt sich die Silvesterkapelle mit dieser Bildgestalt also unverkennbar in die Reihe der Stationskirchen und sie spiegelt darin ihren Entstehungszusammenhang: Vertreterin der großen Basiliken des Papsttums zu sein.

Die meisten Tondi sind allerdings heute bis auf den unteren Saum zerstört. ${ }^{25}$ Auf der Südseite der Kapelle ist heute überhaupt nur noch ein Tondo hinlänglich erhalten,

23 Der Durchmesser der Büstenmedaillons beträgt für das Innenfeld rund $52 \mathrm{~cm}$ und insgesamt mit Rahmung $66 \mathrm{~cm}$.

24 Mitchell 1980 überging die Tondi bei der Beschreibung des Bestandes an Fresken der Kapelle (S.19, Anm.15) und erwähnte sie erst auf S.31 mit einem Satz im Zuge seiner Überlegungen zu den stilistischen Zusammenhängen des Konstantin-Silvester-Zyklus, was die Totalität des inszenierten Anspruchs nicht deutlich machte. Barberini 1993, 55 sprach nur in einem Halbsatz pauschal von verschieden Prophetenbüsten. Erst Zahlten 1994, Sohn 1997 und Noll 2011 betrachteten die Tondi genauer.

25 Die Gleichartigkeit der Fehlstellen im oberen Bereich der Tondi lässt darauf schließen, dass der Verlust durch eine bauliche Maßnahme nach der Mitte des 17.Jahrhundert verursacht wurde. 
auf der Nordseite sind in recht unterschiedlichem Erhaltungszustand nur noch vier volle Tondi zu sehen. Gezeigt werden jeweils Brustbilder männlicher Personen, die vor der Brust mit ausgebreiteten Händen eine geöffnete Schriftrolle weisen. Innerhalb der Tondi werden beiderseits der Köpfe die Dargestellten durch Inschriften benannt. Auch die Schriftrollen sind beschrieben, bis auf wenige zusammenhanglose Buchstabenreste sind jedoch fast alle vergangen.

Und doch sind Aussagen möglich - und damit kommt das Moment der Schriftlichkeit im Raum zum Tragen: Im Jahre 1637 nämlich fertigte der italienische Maler Antonio Eclissi Nachzeichnungen des Freskenschmucks der Silvesterkapelle an, soweit er damals erhalten war. ${ }^{26}$ Weitere unkolorierte Nachzeichnungen unbekannter Hand, aber ebenfalls des 17. Jahrhunderts, haben sich in der Biblioteca Vaticana in einem aus der Sammlung der Barberini stammenden Heft gefunden. ${ }^{27}$ Beide Nachzeichnungsfolgen aber zeigen nicht nur die heute noch weitgehend oder auch nur bruchstückhaft erhaltenen Tondi in einem vollständigeren Zustand, sondern sie überliefern auch eine größere Zahl von Medaillons, die heute verschwunden sind, und - das ist besonders glücklich - sie bieten für acht Tondi die heute fast gänzlich verlorenen Beschriftungen: neben den Benennungen der Auftretenden auch die Aufschriften auf den von diesen dargebotenen Rotuli. Darüber hinaus gibt das Heft Eclissis auf fol. 2 noch einen Grundriss des Oratoriums mit Angaben zu den Plätzen der Büsten innerhalb der Kapelle, so dass die Raumordnung der Tondi festgelegt ist.

Aber noch mehr: Als ich die Nachzeichnungen Eclissis in Wien überprüfte, konnte ich entdecken, dass über die sorgfältig in schwarzer Tusche gearbeiteten Wiedergaben der acht voll genannten Inschriften hinaus auf den Stellen der wiedergegebenen Rotuli, die dem Kopisten nicht mehr recht lesbar und verständlich waren, in feinstem Bleistift Leseversuche notiert sind: und zwar bei sieben weiteren Schriftbändern. Leider sind sie so fein, dass sie in Abbildungen der Kopien nicht zu erkennen wären. Da der Schreibduktus dem der gesicherten Aufschriften gleicht, dürfte es sich um Versuche Eclissis handeln, verbliebene Buchstabenreste zu fassen. Eclissi hat auf diese Weise Befunde bewahrt, aus denen sich weitere 7 Rotulus-Inschriften erschlie-

26 Zahlten 1994, 19-43. Sohn 1997, 24-27. Die Zeichnungen, angefertigt im Auftrag von Francesco Barberini, bilden ein umfangreicheres Heft, das 1881 aus dem Nachlass Overbecks von der Österreichischen Nationalbibliothek Wien erworben wurde: Codex Series nova 3311; Mazal/Unterkircher 1967, 55. Eine Nachzeichnung der Tiaraübergabe veröffentlichte farbig Mazal 1981, Nr.44, Abb. 3. Noll 2011, 22-27 bildet die Tondi von Abraam, Isaac, Iakob, David, Salomon, Daniel ab. Zu Ecclissi und seinem Vorhaben siehe: De Marchi 1993. Herklotz 2006, bes. 218-221.

27 Die bislang unpublizierten unkolorierten Nachzeichnungen (Bleistift) - einer alten Signatur: Barb 1049 auf fol.45r zufolge aus einem geschlossenen Barberini-Bestand - sind großzügig ausgeschnitten in einen neueren Band (wohl des frühen 19.Jahrhunderts) der vatikanischen Bibliothek eingeklebt: Vat. Lat. 9849 fol.40v bis 52r (außer 43v-44v); bei der Neuzusammenstellung wurde die ursprüngliche Reihenfolge der Nachzeichnungen offenkundig zerstört. Beide Folgen der aus stilistischen Gründen ungefähr gleichzeitigen Nachzeichnungen zeigen einen ähnlichen Erhaltungszustand der Fresken, geben bei den Inschriften jedoch unterschiedliche Einzelheiten; dazu im Folgenden. 
ßen lassen. Diese Inschriften auf den Schriftrollen der Brustbilder aber enthalten entscheidende Hinweise, ja den Schlüssel zur Deutung des Gesamtprogramms der Bild-Schrift-Inszenierung in der Silvesterkapelle.

\section{Der durch Nachzeichungen überlieferte, teilweise auch erhaltene Bestand und seine Position im Raum}

Der von Eclissi mit Angaben zu den Orten der Tondi versehene Grundriss erlaubt, die heute nur noch in wenigen Resten erhaltenen Tondi zu benennen und über ihre Position im Raum der Silvesterkapelle zuletzt auch den Sinnzusammenhang ihres Auftretens zu bestimmen, was dann Schlüsse auf den Gehalt der Schrift im Raum der Silvesterkapelle erlaubt.

Neben dem alten (und heute wieder benutzten) Eingang in der Westseite des geosteten Raumes stehen zunächst auf dem südlichen Abschnitt der Westwand die heute nur noch halb erhaltenen - Medaillons von ABRAAM (Eclissi fol.3) und ISAAC (Eclissi fol.4), beide tragen eine unten goldgesäumte Tiara mit Anhängen (candae). ${ }^{28}$

An der folgenden Südseite kam es durch Eingriffe, vor allem durch die Anlage eines neuen großen Eingangs im Jahre 1570 zu erheblichen Verlusten in der Reihe der Büstenmedaillons. Hier fand Eclissi an ISAAC um die Ecke anschließend ein - heute völlig verlorenes - namenloses Medaillon vor, das er mit dem Text des Schriftbandes auf fol. 5 wiedergibt. Neben diesem Tondo unterbricht die tief heruntergezogene Laibungszone eines späteren Fensters die Medaillonreihe; die heute in der Laibung sichtbare einfache Sternblumen-Bemalung weist auf das frühe 14.Jahrhundert hin. So scheint eine nachträgliche Verbreiterung und Vertiefung der Laibung an dieser Stelle schon früh Medaillons zerstört haben.

Von den nachfolgenden Medaillons in der Südwand waren offensichtlich schon im 17. Jahrhundert nur noch so geringe Reste vorhanden, dass sie im Eclissi-Codex keine Berücksichtigung finden konnten. Erhalten ist lediglich im Anschluß an einen nach rechts offenen Halbkreis eines ansonsten verlorenen Tondos nahezu in der

28 Die Tiara wird in Ex 28,37-40 und 29,9 als Kopfbedeckung des priesterlichen Aarons und seiner Söhne genannt, die Patriarchen sind also auch als priesterlich angezeigt. Die in Ergänzung zur Beschreibung der Anordnung der Medaillons im Raum genannten Folio-Zahlen geben die Seiten an, auf denen die Nachzeichnung der jeweiligen Büste im Eclissi-Codex erscheint. Zahlten 1994, 24 bietet Abbildungen der Büsten von Abraam und einem unbestimmten Medaillon (fol.3 und 23); Seroux d'Agincourt 1825 hatte auf der Kupferstichtafel CI mit den übrigen Fresken der Kapelle auch sechs Büstenmedaillons (Johel, Ezechias, Amos, Naum, Ezechiel, Isais) abgebildet, siehe Zahlten 1994, Abb. 21. - Bei der Schreibung der Namen folge ich den epigraphischen Befunden und im Übrigen der Vulgata. 


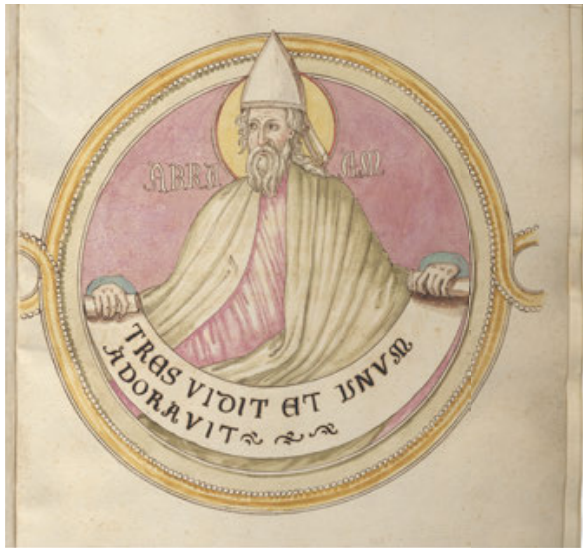

Abb. 9: ABRAAM (Eclissi fol.3).

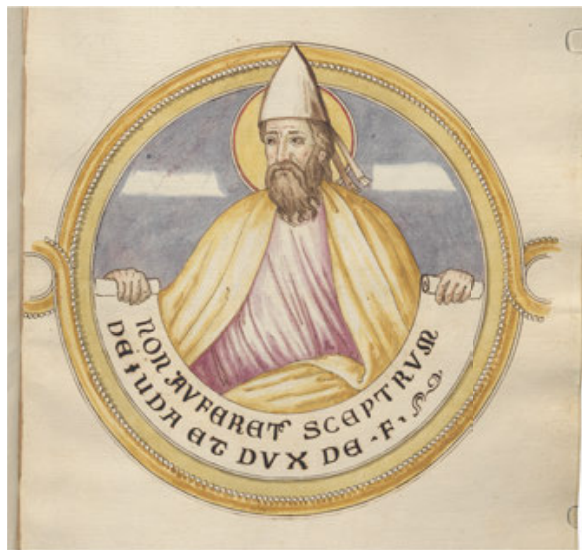

Abb. 11: lacob (Eclissi fol.5).

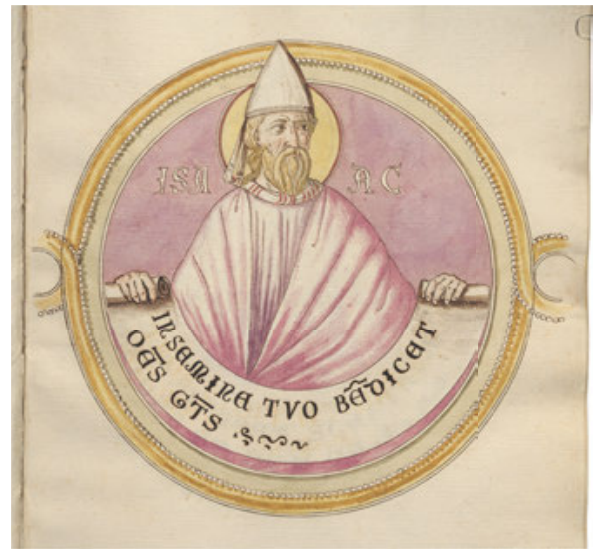

Abb. 10: ISAAC (Eclissi fol.4).

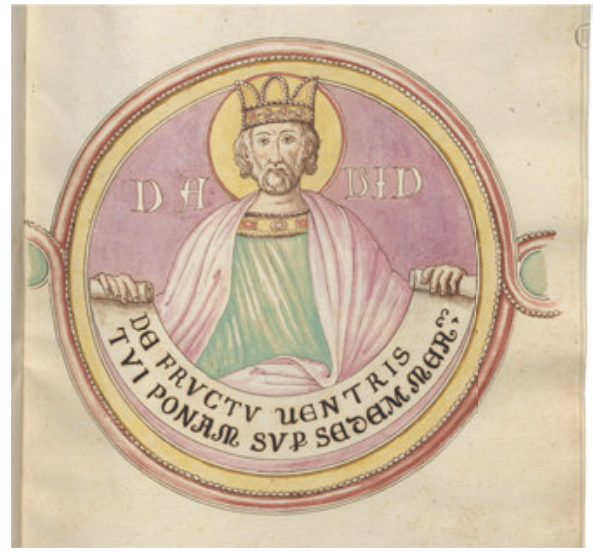

Abb.12: DAVID (Eclissi fol.9).

Mitte der Südwand ein - heute noch vorhandenes - Medaillon, das Eclissis Grundriss als das von SAMUEL bestimmt; ${ }^{29}$ die Nachzeichnung findet sich bei Eclissi auf fol. 6. Danach ist die Medaillonfolge auf der Wand erneut gestört, diesmal durch den Durchbruch des neuen Eingangs im 16.Jahrhundert. Sichtbar ist nur noch ein nach links offener Halbkreis eines Medaillons, diesem dürften - dem verfügbaren Raum nach - zwei weitere Medaillons gefolgt sein. An den Eingang des 16. Jahrhunderts anschließend nennt der Grundriss gegen Ende der Südwand für den dort mit einem Teil des Kopfes noch sichtbaren Tondo den Namen des Königs von Juda EZECHIAS, den der Eclissi-Codex als Ungekrönten auf fol.7 zeigt. Gleich anschließend, vor der Ecke zum Chorraum hin, ist noch ein gleicherweise halb erhaltenes Medaillon

29 Von der Namensbeischrift ist heute nur noch das abschließende L zu lesen. 


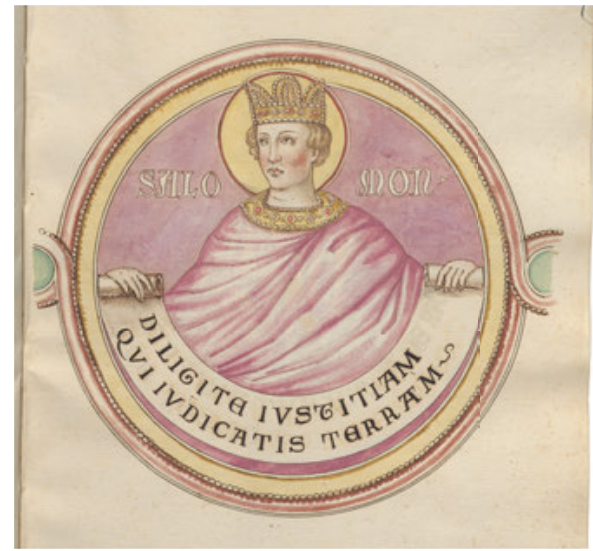

Abb.13: SALOMON (Eclissi fol.10).

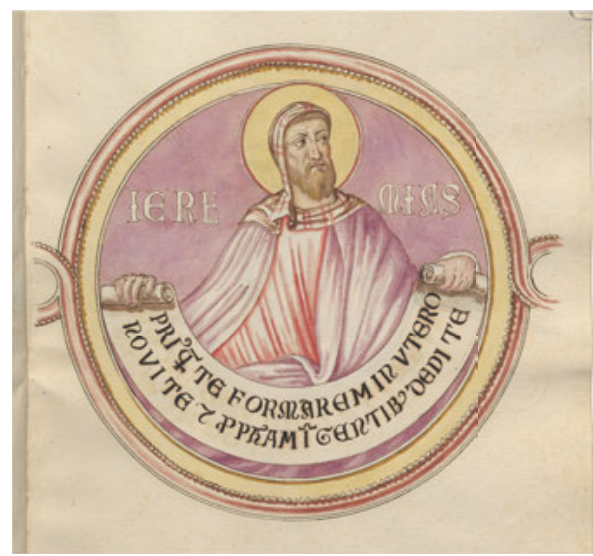

Abb. 15: IEREMIAS (Eclissi fol.12).

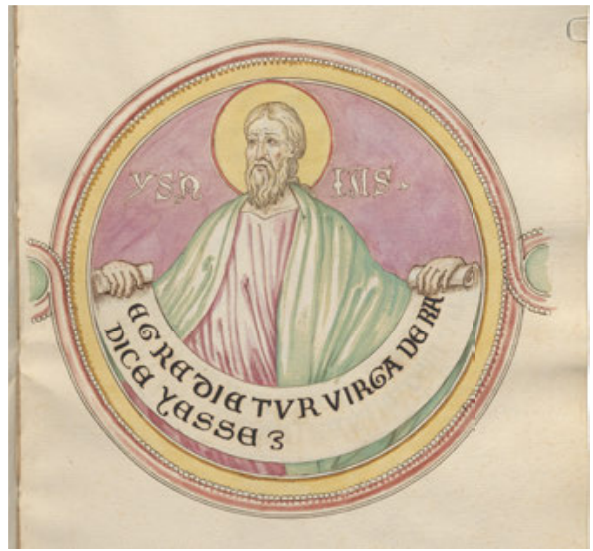

Abb. 14: YSAIAS (Eclissi fol.11).

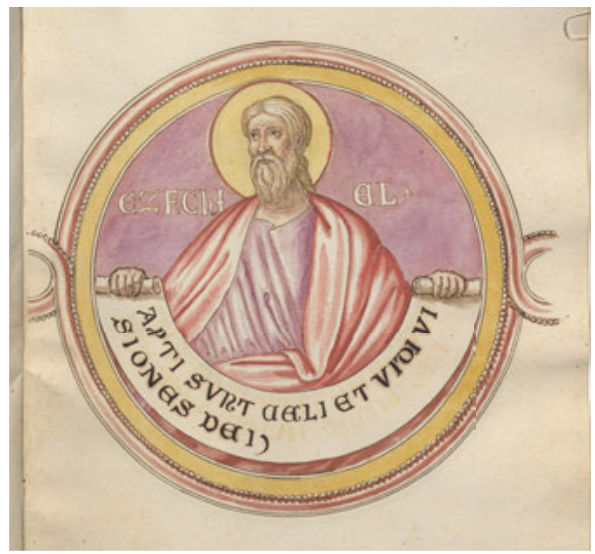

Abb.16: EZECHIEL (Eclissi fol.13).

erkennbar, für das der Grundriss ebenso wie die Nachzeichnung nur das fragmentarische Namensende -AS bieten. Der Maler des 17. Jahrhunderts zeigt in seinem Aquarell eines Gekrönten (fol. 8) das Fehlen des Namensanfangs links neben dem Kopf des Gekrönten durch eine farbfreie Fläche an. Da der voraufgehende Tondo als der des König Ezechias bestimmt ist, dürfte auch der folgende Tondo einen positiven und in der Haltung Ezechias entsprechenden König zuzuweisen sein, und er muss, da die gesamte Reihe offenkundig der Zeitfolge nach angeordnet ist, nach Ezechias regiert haben. Das führt angesichts des von Eclissi und dem Barbarini-Zeichner vorgefundenen Namensrestes -AS auf den in seiner Jahwetreue EZECHIAS gleichenden König JOSIAS. Tatsächlich berichten die Geschichtswerke der Bibel (4 Sam [2 Reg] 22-23; 2 Par [Chron] 34-35) ganz so wie bei Ezechias, dass er tat, was recht war in den Augen von JHWH: Beide Könige unterwarfen sich bedingungslos dem Machtan- 
spruch und dem Gebot von JHWH. Die Gesamtzahl der Medaillons auf der Südseite dürfte - berechnet man eine gleichmäßige Größe der Tondi - insgesamt wohl zwölf betragen haben.

Auf dem nördlichen Abschnitt der Westwand erscheinen zunächst, wie der Grundriss Eclissis überliefert, das Medaillon des gekrönten DAVID (Eclissi fol.9), dann das des ebenfalls gekrönten SALOMON (Eclissi fol.10), so dass die beiden Abschnitte der Westseite zusammen vier Tondi bieten. Sie alle sind nur noch in den unteren Bereichen erhalten. Um die Ecke folgen dann nach den Angaben Eclissis an der Nordseite des Raumes nacheinander YSAIAS (Eclissi fol.11), IEREMIAS (Eclissi fol.12), EZECHIEL (Eclissi fol.13), DANIEL (Eclissi fol.14), OSEAS (Eclissi fol. 15) ${ }^{30}$, JOHEL (Eclissi fol.16), AMOS (Eclissi fol.17), JONAS (Eclissi fol.18), MICHEAS (Eclissi fol.19), NAUM (Eclissi fol.20), ABACHUC (Eclissi fol.21) ${ }^{31}$, SOPHONIAS (Eclissi fol.22) $)^{32}$ - insgesamt also zwölf Medaillons, von denen heute - nach zwei halbzerstörten Tondi - nur noch die mittlere Gruppe von AMOS, IONAS, MICHEAS (unter der Tiara-Übergabe) hinlänglich erhalten ist. Zum Eck hin folgt dann noch nur schemenhaft erkennbar - SOPHONIAS. Den Namensnennungen Eclissis nach orientiert sich diese Folge an der üblichen Reihung der vier Grossen, dann der ersten der 12 Kleinen Propheten, wobei Abdias übersprungen ist und am Ende zwei Propheten weggelassen sind.

Die Ostseite trug zu beiden Seiten des Chorbogens jeweils ein Medaillon. Davon wurde das des Südabschnitts der Wand weitgehend zerstört, als im Zuge des Umbaus von 1570 an Stelle des einst wohl vorhandenen kleinen Chorraums ein neues größeres Presbyterium mit erweitertem Durchgang errichtet wurde. Da aber die Verbreiterung des Bogens nicht streng mittig, sondern - offensichtlich unter Beibehaltung der nördlichen Bogenlaibung des 13. Jahrhunderts - nach Süden verschoben erfolgte, blieb auf dem Nordabschnitt der Ostwand noch umrisshaft ein Medaillon erhalten. Eclissi gibt es auf fol.23r ohne Benennung und Rotulustext wieder. Offensichtlich war die Inschriften stark zerstört und bot nur diffuse Reste, denn der Maler gibt wie bei dem letzten Medaillon der Südseite (-AS für Josias) das Fehlen des Namens durch freie Fläche zu beiden Seiten des Kopfes an. Das Bildnis gleicht weitgehend dem des Sophonias, so muss der Tracht nach erneut ein Prophet gemeint sein.

Weiter führt in diesem Falle die Barberini-Nachzeichnung, die die vatikanische Sammlung auf fol.51v zeigt: Sie gibt ein OSE als Namensbeginn. Der einzige

30 Diese Zeichnung ließ Eclissi als einzige unaquarelliert.

31 Zwischen den Angaben im Grundriss auf fol. 2 und der Bilderfolge im Codex fol.4-23 bestehen an dieser Stelle Unstimmigkeiten; der Grundriss nennt auf der Nordseite nach dem Namen NAUM als letzten der Reihe allein noch den Namen SOPHONIAS, während die Bilderfolge zwischen NAUM auf fol. 20 und SOPHONIAS auf fol. 22 und auf fol. 21 noch ABACHUC zeigt.

32 Wie die Linie durch das Medaillon des Joel zeigt, muss auch bei den Nachzeichnungen mit Ergänzungen gerechnet werden. Offensichtlich für eine spätere Ergänzung sind in der Nachzeichnung des Medaillon -AS Partien frei geblieben. 
Prophet jedoch, dessen Name so beginnt, Oseas, kann nicht gemeint sein, er tritt ja schon - Eclissis Angaben zufolge - vier Medaillons zuvor auf der Nordwand auf. Da das Fresco aber, wie die sorgfältige Wiedergabe Eclissis anzeigt, an dieser Stelle stark schadhaft war, dürfte es sich bei diesem OSE um einen abgebrochenen Leseversuch des Barberini-Zeichners handeln. Die Buchstabenfügungen dieses Zeichners müssen demnach gleichsam als Formangaben verstanden und nur in ihren groben Umrissen und Formeigenschaften für die Deutung berücksichtigt werden: Nach Maßgabe des Schriftduktus der übrigen Namensbeischriften muss es sich bei den ersten drei Buchstaben des Unbenannten um solche gehandelt haben, die (möglicherweise auch in unzialer Schreibung) zu größeren Teilen rund waren oder doch so aufgefasst werden konnten. Dabei dürften, wie die Lesung als S und E nahelegt, beim zweiten und dritten Buchstaben im mittleren Bereich eine Art von waagrechtem Querstrich zu sehen gewesen sein. Diese Merkmale aber treffen neben S und dem (unzial) rund geschriebenen $\mathrm{E}$ auch für das ebenfalls ganz rund geschriebene $\mathrm{G} \mathrm{zu} .^{33}$ Der erste Buchstabe, von dem, wie die Deutung des Zeichners als 0 zeigt, offensichtlich nur runde Teile erhalten waren, sind als weitere Lesungen $M$ und A möglich, die beide Nachzeichner überwiegend in unzialer Schreibung mit starken Rundungen wiedergeben. Da nun das Kopftuch den Dargestellten ikonographisch auf einen Propheten festlegt, wodurch er ganz zwanglos an die Reihe der Propheten vor ihm anschließt, bleiben, weil - bis auf den übersprungenen Abdias - alle übrigen,Kleinen Propheten“ schon zuvor aufgetreten sind, für den fragmentarisch erhaltenen Tondo nur Aggeus, Zacharias und Malachias übrig. Von den Genanten aber bleibt nach der Formen der Buchstaben nur AGGEUS (oder AGEUS). ${ }^{34}$ Allein sein Name verfügt - wenn man wie in mehreren anderen Aufschriften ein unziales A (und E) zu Grunde legt - über drei Anfangsbuchstaben, die der Maler mit Rundungen wiedergeben konnte. ${ }^{35}$

Schon aus der Auswahl der Dargestellten ergeben sich erste Hinweise auf die Absichten der Planer des Zyklus. Es treten durchweg normsetzende Vertreter des Alten Bundes auf, und zwar, wie die Wahl von Ezechias und Josias zeigt, solche deren Wirken mit der Durchsetzung der Gebote von JHWH verbunden ist.

33 Vgl. etwa die Buchstaben E G und S in dem Schriftband des ISAAC.

34 Bemerkenswert ist, dass Aggeus als Büstenmedaillon mit beschrifteten Rotulus auch in San Nicola in Carcere erscheint (um 1128); dazu unten.

35 So ist unwahrscheinlich, dass der nach Amos übersprungene Abdias (Obadja) - mit dem teilweise eckigen B an zweiter Stelle - hier gegen die übliche Reihung doch noch auftrat. 


\section{Die Texte der Schriftbänder in den Tondi und ihr Aussageziel}

Bei keinem der Schriftbänder in den Tondi sind heute an der Wand mehr als wenige Buchstabenreste der Aufschriften erhalten. Doch erneut bieten hierfür die Nachzeichnungen Hilfe, wenngleich Eclissi nur bei sieben Medaillons über die Namen der Dargestellten hinaus auch den Text des durchweg zweiteiligen Schriftbands überliefert es sind dies:
ABRAAM: TRES VIDIT ET UNUM / ADORAVIT;
ISAAC: IN SEMINE TUO BENEDICET / OMNES GENTES; ${ }^{36}$
DAVID: DE FRUCTU VENTRIS / TUI PONAM SUPER SEDEM MEAM;
SALOMON: DILIGITE IUSTITIAM / QUI IUDICATIS TERRAM;
YSAIAS: EGREDIETUR VIRGA DE RA / DICE YESSE;
IEREMIAS: PRIUSQUAM TE FORMAREM IN UTERO / NOVI TE ET PROPHETAM IN GEN- TIBUS DEDI TE; ${ }^{37}$
EZECHIEL: APERTI SUNT CELI ET VIDI VI / SIONES DEI;

Hinzu kommt das bei Eclissi namenlose Medaillon am Beginn der Südseite: Denn der Satz des Schriftbandes, den der Nachzeichner als NON AUFERETUR SCEPTRUM / DE JUDA ET DUX DE F[ermore] wiedergibt, ${ }^{38}$ ist der Beginn von Jakobs Segen für Juda in Gen 49,10: Non auferetur sceptrum de Juda, et dux de femore eius, donec veniat qui mittendus est, et ipse erit exspectatio gentium; „Nicht genommen wird das Szepter von Juda, noch die Führerschaft von seiner Lende, bis der Gesandte kommt, und er wird die Erwartung der Völker sein.“ Dargestellt ist also Iacob. Die Gestalt des Dargestellten bestätigt diese Bestimmung, denn wie ABRAAM und ISAAC trägt auch Iacob als der dritte der Patriarchen eine Tiara.

Die Spezifik der vollständig überlieferten Schriftbandtexte lässt eine mit der Funktion der Medaillons im Bildensemble der Silvesterkapelle zusammenhängende besondere Aussageabsicht vermuten, was eine eingehendere Betrachtung ihres Gehalts notwendig macht. Der Text des ABRAAM-Bandes: TRES VIDIT ET UNUM ADORAVIT stellt kein unmittelbares Schriftzitat dar, sondern fasst in knapper Fügung die Begegnung Abrahams mit den drei Besuchern bei der Terebinthe von Mamre, die in Gen 18,1-8 geschildert wird. Im bereitwilligen Dienst des Stammvaters gegenüber den Drei sah die Kirche die Huldigung und Anerkennung der Herrschaft Gottes dargestellt und darüber hinaus auch die Dreigestalt der von Abraham verehrten göttli-

36 Die Barberini-Nachzeichnung bietet nur die erste Zeile, es fehlt also omnes gentes.

37 Schreibkürzel der Inschriften (etwa bei prophetam bei Ieremias) sind hier und im Folgenden grundsätzlich aufgelöst; Ergänzungen des Verfassers stehen in eckigen Klammern.

38 Das liegende kürzelartige Ornament (wohl für „sequentes“) zeigt, dass das nachfolgende mitangesprochen ist. - Die Übersetzungen der biblischen Texten stammen von mir, auf der Grundlage der Vulgata. 
chen Wesenheit. Der Text des Rotulus von ISAAC, IN SEMINE TUO BENEDICET OMNES GENTES, ${ }^{39}$ ist eine Abwandlung von Gottes Botschaft an Abraham und Isaac Gen 22,18 (Et benedicentur in semine tuo omnes gentes terrae, quia obedisti voci meae). In ihr wird der Patriarch als Quellgrund des kommenden Segens der Völker bezeichnet, und dies - wie die implizit mitausgesprochene Fortführung des Bibelverses besagt - im Hinblick auf seinen Gehorsam gegenüber Gott. Das Schriftband Iacobs, des dritten Patriarchen, die Umformulierung des Segens für Juda in Gen 49,10, spricht vorausweisend David, den ersten König aus dem Stamme Juda an. Hinter diesem jedoch scheint, wie die nun auf Gott bezogene Pluralformulierung zeigt, der Jacobund Davidnachkomme Jesus auf. Ihm wird die Herrschaft zugewiesen und auch das kriegerisch-weltliche Dukat, das den Kern des Imperatorentums ausmacht. Er also ist der Fluchtpunkt der Aussagen.

Bestätigt wird das durch den Vers Davids: DE FRUCTU VENTRIS TUI PONAM SUPER SEDEM MEAM. Er ist Psalm 131 entnommen, der jedoch in Vers 11 sedem tuam sagt ${ }^{40}$. Es handelt sich um die Wiedergabe eines Gotteswortes; der Psalmist, als der dem Mittelalter ja grundsätzlich David gilt, preist den Schwur des Herrn, einen Nachkommen Davids auf Davids Thron zu setzen. Im Fresco ist der Psalmenvers 11 also umformuliert: Durch die Veränderung des sedem tuam des Psalms in das sedem meam der Inschrift verändert der Frescomaler den Text freilich erheblich. Denn da tui nach wie vor David, den Sänger des Psalms, als angesprochene Person erweist, muss sich das meam auf den vom Sänger mit seinem Schwur zitierten Gott beziehen. Damit aber gilt die Herrschaftskündung dem Davidnachkommen Jesus, der den Thron des Göttlichen Vaters besitzen wird.

Das Schriftband von SALOMON bietet den ersten Vers des Buchs der Weisheit (Sap.1,1), als dessen Verfasser dem Mittelalter Salomon galt: DILIGITE IUSTITIAM QUI IUDICATIS TERRAM. Gerade dieses Zitieren des Buchbeginns will nun zweifellos nach Maßgabe mittelalterlicher Zitiergewohnheit die nachfolgenden Verse in die Aussage einbezogen wissen - gleichsam als mitausgesprochen. ${ }^{41}$ Und diese anschließenden Verse vereindeutigen die Aussage des zitierten ersten Verses in Richtung einer unmittelbaren Beziehung jeglicher Herrschaft auf Gott: „(1) Liebet die Gerechtigkeit, die ihr die Erde richtet! Denket über den Herrn in rechter Gesinnung, und in Einfalt des Herzens suchet ihn! (2) Denn zu finden ist er von denen, die ihn nicht versuchen, er offenbart sich aber denen, die Vertrauen haben in ihn. (3) Verkehrte Gedanken nämlich trennen von Gott, aber auf die Probe gestellt verdirbt die Macht die Toren.“ Mit dem Richteramt, der vornehmsten Pflicht des Königtums, wird somit die Herrschaft ganz allgemein umfassend von der rechten Bezogenheit zu Gott abhängig

39 Von dem Text ist S.MI.. T (semine tuo) an der Wand noch zu lesen.

40 Ps. 131,11: Juravit Dominus David veritatem, et non frustrabitur eam: de fructu ventris tui ponam super sedem tuam.

$41 \mathrm{Zu}$ solchen komplexen ,Hintergrundzitaten“ vgl. Berschin 1980, 299-309; bes. 303ff.; vgl. dazu auch Schneider 1991, 226-258; 253f. mit Anm. 68. 
gemacht und der dagegen Verstoßende als der göttlichen Strafe ausgeliefert festgestellt.

Die Worte des auf der Nordseite anschließenden Medaillons des YSAIAS sind geeignet, diese Gedanken in positiver Richtung weiter zu führen. Das Schriftband zitiert mit Is 11,1: EGREDIETUR VIRGA DE RADICE YESSE, ,ausgehen wird von der Wurzel Jesse das Reis“, den Beginn der Beschreibung der Herrschaft des von Gott Erwählten aus Jesses und Davids Geschlecht. Auch hier will das Zitat des ersten Verses zweifellos die nachfolgenden Verse aufrufen, die erst den hier gemeinten Aspekt der virga erläutern: „(2) Und ruhen wird der Geist des Herrn über ihm, der Geist der Weisheit und des Verstandes, der Geist des Rates und der Tapferkeit, der Geist der Erkenntnis und der Frömmigkeit. (3) Und erfüllen wird ihn der Geist der Furcht des Herrn: Nicht nach dem Augenschein wird er richten, noch nach dem Hörensagen urteilen. (4) Sondern in Gerechtigkeit wird er richten die Geringen und in Billigkeit für die Sanftmütigen der Erde urteilen. Die [Gewalttätigen der] ${ }^{42}$ Erde wird er schlagen mit dem Reis (virga) seines Mundes und mit dem Hauch seiner Lippen töten den Frevler“. Gemeint ist mit virga somit nicht nur der nachgeborene ,Spross“, sondern zugleich die strafende ,Rute‘: Angesprochen ist also eine zukünftige gottgefällige Herrschaft des Jesse-Nachfahren in ihrem strafenden Aspekt.

Der Text der Schriftrolle des IEREMIAS: PRIUSQUAM TE FORMAREM IN UTERO NOVI TE ET PROPHETAM IN GENTIBUS DEDI TE gibt verkürzt den Vers Jer 1,5, mit dem der eigentliche Text des Jeremias einsetzt: „Bevor ich dich bildete im Mutterleib kannte ich Dich (und bevor du hervorgingst aus dem Schoß heiligte ich Dich) und habe Dich bei den Völkern zum Propheten bestellt.“ Auch hier muss der ganze mit den zitierten Worten eingeleitete Vorgang wahrgenommen werden. Denn nach der Widerrede des so Bestellten und deren Zurückweisung durch Gott (1,6-9) wird im Vers 10 die herrscherliche Einsetzung des Gottgesandten ausgesprochen: „Siehe ich setze dich heute über die Völker und über die Königtümer, dass du ausreißest und vertilgst und zerstörest und aufbauest und pflanzest." Damit wird erneut das herrscherliche Gericht Gottes angekündigt, als dessen Mitwisser und Vollzieher der eingesetzte Gesandte Gottes bestellt ist. Obwohl im Ursprungstext zunächst Jeremias angesprochen ist, zielt der Aufruf des Verses hier doch - im Sinne mittelalterlicher Schriftdeutung - wesentlich auf Jesus und muss vor dem Hintergrund der im Rotulus von ABRAAM angesprochenen christlichen Trinitätslehre gehört werden, als Hinweis auf die Fleischwerdung des göttlichen Sohnes in Maria. Ihm werden die kommende Herrschaft und das Gericht zugewiesen.

Auch die Worte des Rotulus von EZECHIEL: APERTI SUNT CELI ET VIDI VISIONES DEI sind dem Eingangsvers von dessen Buch entnommen (1,1b). Wiede-

42 Die Vulgata bietet hier (im Anschluss an die LXX) nur terram, angesprochen sind aber die den mansuetis Entgegenstehenden; in der ,Jerusalemer Bibel' ist der masoretische Text an dieser Stelle korrigiert. 
rum soll also das Folgende mitgehört werden, und dies ist die mit einem erneuten vidi in 1,4 eingeleitete ${ }^{43}$ große Vision des Ezechiel von Gottes machtvollem Thronen auf dem von den vier Animalia - den vier Evangelisten nach kirchlicher Lehre - getragenen elektrongleißenden Feuerthron (1,5-25). Aufgerufen ist mit diesem Vers also nicht so sehr die Berufung des Propheten, als vielmehr die Erscheinung Gottes $(1,26-$ 28) und das von ihm ausgehende Strafgericht über die Abtrünnigen und die gegen Gottes Willen Verhärteten und Widerspenstigen (2,3-4).

In allen Fällen bieten die Schriftbänder also eine Aussage des Dargestellten, die als Verkündung von Gottes Macht und richterlichem Tun und dann auch als Herrschaftsprädikation des göttlichen Davidnachkommen zu deuten ist.

\section{Die Fragmente der Rotulus-Inschriften nach den Leseversuchen Eclissis}

Die eben erläuterten Inschriften finden eine Ergänzung durch die neu entdeckten Leseversuche Eclissis von einzelnen Wörtern oder Buchstabengruppen, und zwar bei den Rotuli von DANIEL, SAMUEL, AMOS, JONAS, MICHEAS. Zwar sind diese Bleistiftangaben für den heutigen Leser nur mit großer Mühe zu lesen, so dass zuweilen Unsicherheiten bleiben. ${ }^{44}$ Gleichwohl sind die Reste in drei Fällen, bei AMOS, JONAS und MICHEAS, so umfangreich, dass die Aufschriften eindeutig bestimmt werden können.

Auf dem Schriftband des IONAS ist in einer ersten Zeile am Beginn neben der Hand SICUT IONAS notiert; nach einer größeren Lücke sind zwei annähernd senkrechte Striche zu erkennen, denen ein ENT folgt. Die zweite Zeile beginnt mit einem T, dem ein T-ähnlicher Buchstabe folgt. Nach einer kleineren Pause folgt IEBUS, dann E, dem mehrere Buchstaben mit einem (annähernd) senkrechten Strich folgen. Die Buchstabenfolge IEBUS in der zweiten Zeile, die zweifellos zu DIEBUS zu ergänzen ist, gibt den ersten Hinweis, sie besagt zusammen mit dem $\mathrm{T}$ am Zeilenanfang, dass hier an tribus diebus von Jonas 2,1 zu denken ist. Allerdings kann die Aufschrift, wie

43 Vers 2 und 3a stellen eine zweite Einleitung in der 3.Person dar; das vidi von 1,4 ist also letztlich das des Verses 1,1.

44 Zusätzliche Schwierigkeiten ergeben sich dadurch, dass im erhaltenen Bestand mehrfach Ligaturen, Kürzel und Abkürzungen erscheinen und daher auch bei den fragmentierten Inschriften vorgekommen sein werden. Auch werden einzelne Buchstaben auf mehrere Weisen (Antiqua und Unziale) wiedergegeben, etwa E, A, D, V, T, N und M, was die Zuordnung der angezeigten Reste zu bestimmten Buchstaben erschwert. Schließlich finden sich wiederholt Ornamente an den Versenden, deren Reste sich von denen der Buchstaben nur schwer scheiden lassen, zumal wenn Buchstaben - wie das letzte M im Rotulus Davids - über der Zeile geschrieben werden. Bei meinen Deutungen dieser unsicheren Befunde muss also - abgesehen von den Fällen, wo der Befund sehr dicht mit einem Schriftzitat zusammenstimmt - ein Rest von Unsicherheit bleiben. 
das anfängliche SICUT IONAS deutlich macht, nicht unmittelbar ein Zitat aus dem Ionas-Text wiedergegeben haben. Dies führt auf einen Text, der seinerseits die Worte aus Ionas anführt: und zwar auf das Matthäus-Evangelium in 12,40: Sicut enim fuit Ionas in ventre ceti tribus diebus et tribus noctibus, sic erit filius hominis in corde terrae tribus diebus et tribus noctibus. „Denn wie Jona drei Tage und drei Nächte im Bauch des Fisches war, so wird sein der Menschensohn im Herzen der Erde drei Tage und drei Nächte.“ Wohl unter Auslassung von et tribus noctibus und vielleicht ohne Wiederholung der Zeitbestimmung am Versschluss - dürfte das Schriftband gelautet haben: SICUT IONAS FUIT IN VENTRE CETI / TRIBUS DIEBUS, SIC ERIT FILIUS HOMINIS IN CORDE TERRAE (tribus diebus). Die beiden senkrechten Striche in der ersten Zeile sind also die Reste eines V, das mit ENT zusammen VENT [RE] bildet. Das E nach -IEBUS dürfte mit einem der nachfolgenden Striche als ET, als Kürzel für erit aufzufassen sein. Die übrigen senkrechten Striche in der zweiten Zeile müssen die Reste von filius [hominis] $\operatorname{sein}^{45}$, dem dann in dem von Eclissi nicht mehr erfassten Bereich in corde terrae gefolgt sein muss. In der von Matthäus beschriebenen Szene gibt Jesus, als er im Anschluss an seine Rede von der Rechenschaftspflicht jedes Menschen vor dem jüngsten Gericht von den Pharisäern um ein Zeichen seiner messianischen Macht gebeten wird, als Zeichen an, dass der Menschensohn drei Tage im Herzen der Erde sein werde, so wie Jonas drei Tage im Fischbauch geweilt hatte. Mit einigen Vergleichen weist Jesus dann im Anschluss an Ionas' Bußpredigt in Ninive auf die herrschaftliche Größe des kommenden Richters hin.

Auch auf dem Rotulus des MICHEAS sind in zarter Schrift Leseversuche Eclissis zu erkennen. Die zweizeilige Inschrift scheint mit DE begonnen zu haben, dem nach einer kleinen Lücke ein $S$ folgte, an das ein verhältnismäßig gerade ansetzender Buchstabe anschloss. In der zweiten Zeile findet sich nach einer längeren Lücke ein BUM, dem wiederum nach einer längeren Lücke (in der eine Fügung NI oder MI angedeutet ist) ein DE und HIERUS nachfolgt. Diese Angaben genügen, um den Text des Schriftbandes als das Versende von Micheas 4,2 zu bestimmen: DE SION EGREDIETUR LEX / ET VERBUM DOMINI DE HIERUSALEM. Darin wird von den fremden Völkern gesprochen, die sich - nach der Zerstörung Jerusalems wegen der Sündenverfallenheit der Juden - dem in Jerusalem verehrten Einen Gott anschließen und ihn zu verehren herbeikommen. Nach einem Strafgericht über die widerstrebenden Völker wird Gott dann Frieden schaffen, Schwerter werden zu Pflugscharen umgeschmiedet werden und Speere zu Hacken. Wieder wird also Gesetz und Gericht angesprochen, beide gehen von Gott und ,Jerusalem‘ aus.

Etwas weniger sicher, aber immerhin doch höchst wahrscheinlich ist die Bestimmung eines dritten Spruchbandes, das des AMOS. Dafür gibt Eclissi zwei Zeilen an, doch nur in der ersten Zeile notiert er Reste, die Anhaltspunkte für die Rekonstruktion

45 An einem der Striche scheint ein runder Schnörkel anzusetzen, es könnte das Kürzel für [fil]ius sein. 
der Inschrift bieten. Nach einem recht kurzen Wort, für das Eclissi mehrere senkrechte Striche angibt, ist ein D zu erkennen, dem nach kurzer Lücke die Buchstaben MINUM folgen. In dem recht kurzen Text des Amos aber erscheint diese Buchstabenfügung nur an einer einzigen Stelle: in Vers 9,1; er beginnt mit vidi dominum, um dann fortzufahren: stantem super altare et dixit: Percute cardinem et commoveantur superliminaria, avaritia enim in capite omnium [...]; non erit fuga eis. Die wenigen senkrechten Striche am Beginn der ersten Zeile dürften somit die Reste des VIDI sein. Weil von der zweiten Zeile nur ein Buchstabe angegeben ist, wohl ein A, kann die Ausdehnung des Zitierten oder - da Amos 9,1 ein recht umfangreicher Vers ist - die Art der kürzenden Zusammenfassung nicht weiter bestimmt werden. ${ }^{46}$ Jedenfalls ist durch die Eingangsworte der Inhalt der Aussage von Amos' Rotulus nahezu gesichert: Auszugehen ist demnach von VIDI DOMINUM STANTEM SUPER ALTARE ET DIXIT: PERCUTE CARDINEM ET COMMOVEANTUR SUPERLIMINARIA; NON ERIT FUGA EIS. Vielleicht endete das Zitat aber schon mit superliminaria. Erneut ist ein Gerichtsgeschehen angesprochen: „Ich sah den Herrn über dem Altar stehen und er sprach: schlage an die Schwelle und der Türbalken wird erbeben; Habsucht nämlich wohnt in ihrem Kopf, den Rest von ihnen werde ich in das Schwert stürzen, keine Flucht wird ihnen bleiben; sie werden fliehen und keiner wird gerettet werden von den Fliehenden.“ In den folgenden Versen 9,2-10 wird dieses Strafgericht über das Volk Gottes im Einzelnen ausgemalt, erst Vers 11 bringt den Umschwung: die Ankündigung der Wiederherstellung Jerusalems und der endlichen Errettung Israels (9,12-15), mit der Amos endet.

Mit eingeschränkter Sicherheit lässt sich das zweizeilige Schriftband von DANIEL deuten. Der Text beginnt mit einem CUM, an das eine Buchstabenfolge anschließt, die VERER, VENER oder VEDER gelesen werden kann ${ }^{47}$, worauf Reste zweier Buchstaben (möglicherweise ist einer davon ein T) und DO zu folgen scheinen. In der zweiten Zeile notierte Eclissi einen Befund, der nach einem $\mathrm{AB}$ einen (leicht gebogenen) Strich, dann zwei gegenläufige geschlängelte Striche zeigt, die ein $\mathrm{M}$ zu sein scheinen, daran schließt ein UN oder IN und ein vorne gerundeter Buchstabe sowie TIO. In dieser Zeichenfolge: A B ( M I N ( T I O möchte ich ein ABOMINATIO erkennen. ${ }^{48}$ Dieses Wort tritt bei Daniel drei Mal auf, in keiner dieser Stellen jedoch

\footnotetext{
46 Wie das Schriftband Jacobs zeigt, konnte ein Wort auch mit nur einem Buchstaben angegeben werden. Freilich rief das Zitat für den mittelalterlichen Schriftkenner grundsätzlich den folgenden Kontext auf.

47 In der Unzial-Schrift ähneln R, $\mathrm{N}$ und D einander, und sind bei schlechter Erhaltung kaum zu unterscheiden. Zahlten 1994, 22 liest versuchsweise cum venerit, meint jedoch zugleich „fast unlesbar“. 48 Sohn 1997, 26, Anm. 89, der aber den Wiener Codex nicht selbst in der Hand gehabt hat, ergänzt das „Cum venerit“ von Zahlten zu „Cum venerit sanctus sanctorum cessabit unctio“. Er fasst dies als Verweis auf Dan 9,24 auf, der allerdings völlig anders lautet. Noll 2011, 26 folgt Sohn und liest dann „[...] t [?] .s [...] abit unctio“ unter Verweis auf eine im Mittelalter beliebte an Dan 9,24 anschließende Formulierung in einer pseudoaugustinischen Predigt. Aber das führt vom Bibeltext ab, und um den geht es, wie alle anderen Zitate belegen.
} 
findet sich kurz zuvor ein CUM. ${ }^{49}$ Das spricht dafür, dass der Rotulus Daniels wie der Abrahams und Jonas' kein Daniel-Zitat enthielt, sondern eine Formulierung, die auf andere Weise den Bezug zu Daniel herstellt. Tatsächlich findet sich abominatio in den Evangelien bei Matthäus $(24,15 f$.) und Markus $(13,14)$ als Wort Jesu - und zwar als zitierender Hinweis auf Daniel, wie Matthäus ausdrücklich vermerkt (und nur an dieser einen Stelle wird Daniel im Neuen Testament namentlich genannt). Bei ihnen allen wird die Aussage durch ein Cum videritis eingeleitet. So dürfte kaum ein Zweifel bestehen, dass das Schriftband Daniels in der Silvesterkapelle - ähnlich wie im Falle des Jonas - dieses Daniel zitierende Jesuswort war, wohl wegen der Nennung Daniels (die im Schriftband aber sicherlich herausgestrichen war) in der Fassung von Matthäus (24,15f.): CUM ERGO VIDERITIS ABOMINATIONEM DESOLATIONIS, STANTEM IN LOCO SANCTO, QUI LEGIT INTELLIGAT, TUNC QUI IN IUDAEA SUNT, FUGIANT AD MONTES..$^{50}$ Eclissi hatte bei seinem Leseversuch das erste I von VIDERITIS verfehlt. Die Erstreckung des Zitats ist wiederum nur ungefähr ersichtlich, er dürfte kaum über fugiant ad montes hinausgegangen sein, vielleicht aber endete das Zitat schon bei qui legit, intelligat. ${ }^{51}$ Gleichwohl ist der mittelalterlichen Zitierauffassung entsprechend sicherlich der ganze folgende Wortlaut und der Kontext insgesamt mit angesprochen. Und der ist überaus bezeichnend: „Wenn ihr nun seht das Gräuel der Verwüstung, wovon gesagt ist durch den Propheten Daniel, stehen an heiliger Stätte - merke auf, wer liest! - dann fliehe wer in Judaea ist in die Berge“. Das Danielwort ist Teil der Wiederkunftsrede Jesu angesichts des Tempels in Jerusalem, in der die kommende Zerstörung von Stadt und Heiligtum angekündigt wird als Vorzeichen der letzten Dinge, der Wiederkunft und endzeitlichen Herrschaft Christi.

Schwierig und entsprechend unsicher sind die Gegebenheiten bei SAMUEL. Denn obwohl Eclissi hier mehrere Wörter oder Wortreste andeutet, die im Bestand der Bücher Samuel auch erscheinen, sind diese doch nicht eindeutig und fügen sich nicht einfach in den Kontext der übrigen Schriftbänder ein. So ist nicht auszuschließen, dass Eclissis Deutungsversuch hier fehlging. Nach einem längeren Abschnitt (ungefähr sieben Buchstaben lang), in dem Eclissi mehrere nicht recht bestimmbare Buchstabenreste (oft Rundformen) gibt, scheint LACEBAT zu folgen, dann nach einer senkrechten Wellenlinie vielleicht ein DO, ein ET und möglicherweise ein HO. Eine

49 Dan. 9,27; 11,31; 12,11 (nur hier steht ein cum, doch sieben Worte vor abominatio). Alle drei Stellen schildern ein Handeln von Israel feindlichen Fürsten. Andere Wörter vom Stamm abomin- erscheinen bei Daniel nicht.

50 Die Aussage bei Mk13,14: Cum ergo videritis abominationem desolationis, stantem ubi non debeat, qui legit intelligat, tunc qui in Iudaea sunt, fugiant in montes; ähnlich die Aussage bei Lk 20: Cum ergo videritis circumdari ab exercitu Ierusalem, tunc scitote quia appropinquavit desolatio eius.

51 Die Raumverhältnisse auf dem Schriftband könnten nahelegen, dass desolationis gegenüber abominatio vorgezogen war. Wie der Text des Schriftbandes von Iacob zeigt, konnten spätere Worte eines Zitats auch nur durch den Anfangsbuchstaben angezeigt werden. 
solche Folge von Wortresten (wenn sie denn richtig gelesen sind) findet sich tatsächlich in den Büchern Samuels: im ersten Buch Samuel 2,26: ... et placebat tam Domino quam hominibus..$^{52}$ Bei dieser Aussage handelt es sich zwar um eine Äußerung über Samuel; doch findet sich eine ähnliche Aussage bei Lukas 2,52 über Jesus, und zwar als Schlusssatz der Kindheitsgeschichte. So scheint es, dass die Worte von 1 Sam. 2,26 von den Planern der Ausmalung, wie häufig im Mittelalter und so schon im Tondo des Jeremias, im Sinne einer engen Interpretatio ad litteram unter Hintanstellung des ursprünglichen Aussagezusammenhangs, eingeschränkt wörtlich verstanden und wesentlich auf Christus bezogen wurden: Er vermittelt zwischen Gott und Menschen, und eben dies beansprucht der, der sich - den Kaiser verdrängend - als Vicarius Christi sieht. Und zugeordnet ist diese Aussage dem Manne, der mit der Salbung Sauls das alttestamentliche Königtum begründete.

Die Buchstabenreste der beiden letzten Schriftbänder, für die Eclissi Leseversuche überliefert, sind wenig umfangreich, dementsprechend lassen sich die gemeinten Schriftstellen nur mit Vorbehalt umreißen. Für die zweizeilige Aufschrift des JOSIAS [den Tondo -AS] gibt Eclissi als Beginn der ersten Zeile zwei Buchstabenreste, die EC oder EG lauten könnten, dann noch einmal zwei, die AC oder AQ oder AU oder AD (das D unzial geschrieben) meinen könnten. Da die Abschnitte, in denen die Bibel über Josias berichtet, nicht allzu umfangreich sind, es sind im 2. Buch der Könige 22,1-23,30 (und die Parallele dazu im 2. Buch der Chronik 34,1-35,27) und darin nur wenige Äußerungen stehen, die nicht unmittelbar nur Geschichtliches überliefern, kann doch eine begründete Vermutung vorgelegt werden. ${ }^{53}$ Eine kurzes mit E beginnendes Wort, dem seinerseits ein mit AC oder AQ oder AU oder AD einsetzendes Wort nachfolgt, erscheint im Bericht über Josias nur an zwei Stellen: in 4 Reg [2 Reg] 22,11: et audisset rex verba libri legis Domini, scindit vestimenta sua; und in 4 Reg [2 Reg] 22,16f.: ego adducam malam super istum locum et super habitatores eius. Da auch eine Schreibung von quod mit Kürzel möglich ist, ist noch an 4 Reg [2 Reg] 22,18f. zu denken: pro eo quod audisti verba voluminis [i. e. Biblia ${ }^{54}$ ] et perterritum est cor tuum [...] et ego audivi. Allen diesen Texten (mit ihren Paralleltexten im 2. Buch Par [Chron] ${ }^{55}$ ist das Richterlich-Verurteilende, verbunden mit einer Gehorsamsbereitschaft des Königs und Gottes Eingehen darauf, gemeinsam, und dies vermute ich als Aussage des verlorenen Schriftbandes. Wegen der besonderen Prägnanz ist vielleicht 4 Reg [2 Reg] 22,16f. als Grundlage der Formulierung des Schriftbandes zu bevorzugen: ego adducam malam super istum locum et super habitatores eius.

52 Den Buchstabenresten zufolge müsste das tam ... quam durch et ersetzt worden sein, das auch bei Lukas steht.

53 Nicht völlig auszuschließen ist aber auch zwei senkrechte Wellenlinien zu erkennen, die dann als M zu interpretieren wären, was auf die Aussage Josias' in 4 Reg [2 Reg] 22,13 führen würde: magna enim ira Domini succensa est contra nos, quia non audierunt patres nostri verba huius libri [i. e. sua].

54 Das verba voluminis wäre freilich zweifellos durch verba mea ersetzt worden.

552 Par [Chr] 34,19; 34,24f. 
Vom Schriftband des Abachuc scheinen nur wenige Reste erkennbar gewesen $\mathrm{zu}$ sein, eine Buchstabenfolge A B G oder A B S und später vielleicht T. Sie findet sich in dem nicht allzu umfangreichen Text des Propheten allein in dem Wort abscondita, das nur in Vers 3,4 im Zuge einer Beschreibung von Gottes machtvollem Wirken erscheint: Splendor eius ut lux erit, cornua in manibus eius; ibi ABSCONDITA est fortitudo eius, „Sein Glanz wird sein wie Licht; Strahlen in seinen Händen; darin verborgen war seine Macht.“ Die nachfolgenden Verse würdigen die - auch todbringende - Macht Gottes und seines Heiligen. Als Vorankündigung Christi ist Vers 3,4 für die Kirche eine der wichtigsten Aussagen Habakuks, er wurde auch im Breviarium monasticum zur Lesung vorgesehen ${ }^{56}$ und muss demgemäß allgemein bekannt gewesen sein. So wäre seine Verwendung als Schriftband-Text nicht überraschend, die Formulierung im Einzelnen aber bleibt offen.

\section{Das Auswahl- und Reihungsprinzip der Medaillons}

Der volle Sinn des Auftretens der Dargestellten mit ihren Aussagen ergibt sich freilich erst aus den Prinzipien, nach denen die einzelnen Personen ausgewählt und dann im Raum angeordnet sind. ${ }^{57}$ Eine rein zeitlich begründete Auswahl und Anordnung der biblischen Büsten kann nicht vorliegen, da in diesem Falle die Fortführung der von Abraham zu Isaak und (auf der Südwand) zu Jakob absteigenden Zeitfolge die Stellung Davids und Salomons zwischen Samuel und Ezechias erfordert hätte. Auch eine sonst häufige - Gruppierung der Büsten nach Genera, wie sie die Dreiergruppe der Partriarchen und die Folge der Propheten nahelegen könnte, ist ausgeschlossen, da die in diesem Falle erwartbare ,Königsgruppe‘ zerteilt und historisch verkehrt aufgetreten wäre, auf Westwand und Südwand. Die Annahme zweier Königsgruppen aber, eine der Gesamtherrscher und eine von Königen der späteren Teilstaaten (Ezechias, Iosias) würde unbedingt das Auftreten von Saul verlangen, für den jedoch neben David und Salomon kein Raum ist. Schließlich spricht auch die Folge der Kleinen Propheten gegen eine schlichte Gattungsanordnung, denn da in ihrer Reihe Abdias übersprungen wird, war die Zwölfzahl bei ihnen offenkundig nicht vollständig.

Der Anordnung muss also ein anderes Prinzip zugrunde liegen. Die Gruppe der Kleinen Propheten gibt den entscheidenden Hinweis: Die Kleinen Propheten folgen einander in der Reihung, wie die Vulgata ihre Schriften bietet. ${ }^{58}$ Und dies ist tatsächlich das Reihungsprinzip der Büsten insgesamt: Ausgehend von ABRAAM auf der Westwand und über die Südwand voranschreitend bis hin zu Josias, dann auf der

56 Vorgesehen in der Matutin in der 3. Nokturn in vanis festis.

57 Zahlten 1994, 30 erwägt mehrere Möglichkeiten, ohne abschließendes Urteil. Sohn 1997 und Noll 2011 übergehen diese zentrale Frage.

58 Abgesehen von dem übersprungenen Abdias. 
Nordhälfte der Westwand mit DAVID erneut einsetzend und über die Nordwand bis zu Aggeus und zum Chor hinführend durchziehen den Raum die Büsten der maßgebenden Personen aus der Geschichte des Volkes Israel in der Folge, in der sie mit ihren Büchern (oder Aussagen) im Alten Testament nach dem Text der Vulgata auftreten. So lässt sich das Erscheinen von David und Salomon zwischen Josias und den Propheten erklären, beide gelten dem Entwerfer der Freskenausstattung hier nicht so sehr als Könige, sondern als Verfasser der ihnen zugewiesenen deuterokanonischen Bücher der Schrift, die eben den chronikalischen Büchern des Alten Testamens nachfolgen. Damit bilden die Tondi ein Sinnganzes: Die von ihnen dargebotenen Schriftbänder spiegeln den Kanon der Bibel im Raum - und deuten so die über ihnen hinziehenden szenischen Bilder in ganz spezifischer Weise.

Die Erkenntnis der Auswahl- und Anordnungsprinzipien erlaubt nun auch Vermutungen über die verlorenen Büsten: die Personen, die zwischen den gesicherten Büsten auftraten. Denn angesichts ihres gemeinsamen und gleichartigen Auftretens in einem Sakralraum müssen die fraglichen Personen vergleichbaren Charakters gewesen sein, also gleicherweise Bücher der Bibel repräsentieren - und zwar, wie die überlieferten oder in Resten erhaltenen Schriftbandtexte erweisen, die sämtlich von Gottes Herrschaft und Gericht sprechen, positiven Charakters hinsichtlich der Vorstellungen und Ansprüche einer gottverbundenen Herrschaft. Das bedeutet, dass negative Personen, seien sie auch ranghoch, ausgeschlossen sind, wie etwa Osias, für den Zahlten den Platz nach Ezechias in Erwägung zog. ${ }^{59}$ Denn der Wertung der Schrift zufolge tat er, „was schlecht ist in den Augen Jahwes“60. Das gleiche gilt für Saul, der von Gott verlassen wurde, und von dem schwachen und anmaßenden Roboam sowie von Jeroboam, der nicht der davidischen Familie entstammte, die gleicherweise erwogen wurden.

59 Abgesehen von Jakob, dem nun aufgrund des von Eclissi überlieferten Text zweifelsfrei das erste Medaillon der Südwand zugewiesen ist, zieht Zahlten 1994, 30 als ,Inhaber' verlorener Tondi in Erwägung: zur Komplettierung der Zwölfzahl der ,Kleinen Propheten': Abdias, Aggeus, Malachias und Zacharias (auf Grund der Barberini-Nachzeichnung ist nun der namenlose Prophet des Nordteils der Ostwand als Aggeus bestimmt); zur Vervollständigung der Zahl der Richter: Gideon, Jephta und Samson; zur Ergänzung der ersten Könige Israels: Saul und für die spätere Zeit als Gegenstück zu Ezechias Osias (für den der Namensrest -AS erwogen wird) und darüber hinaus möglicherweise noch Roboam und Jeroboam; schließlich aus der Zeit des Freiheitskampfs gegen die Seleukiden noch Judas Maccabäus und sein Bruder Jonathan.

604 Reg [2 Reg] 17,1f. Dass im politischen Kampf des 13.Jahrhunderts Osias als negative Person galt, belegt das von päpstlicher Seite gegen Friedrich II. in Lyon gefertigte Schriftstück vom Anfang Juli 1245, in dem der Kaiser von der maßlosen Geistlichkeit unter die notorischen Gottesfeinde eingereiht wird, wobei nach dem Pharao, nach Nero, Julian, Nemroth, nach Nachor, Doech und Oza auch Osias auftritt; Winkelmann 1885, 714 Nr. 1037. 


\section{Die Ordnung der Medaillons mit ihren Texten im Raum}

Die Folge nimmt ihren Anfang im Südteil der Westwand mit den drei Patriarchen des umfangreichen Buches Genesis, mit ABRAAM, dem Erzvater des Volkes Gottes, sowie ISAAC und IACOB. Mit diesem springt die Medaillonsreihe auf die Südwand über, wo die Anzahl der Medaillons wegen der Störungen durch die Fensterlaibung und den Durchbruch des Eingangs im 16.Jahrhundert unklar bleibt - aber in Entsprechung zur Nordwand wohl 12 Medaillions anzunehmen sind. ${ }^{61} \mathrm{Im}$ Anschluss an IACOB bietet nach zwei durch die später heruntergezogene Festerleibung zerstörten und weiteren drei verlorenen Medaillons erst SAMUEL wieder einen festen Punkt, er steht für die beiden nach ihm benannten Bücher der Schrift. Dazwischen müssen zunächst die Personen für die übrigen Bücher des Pentateuch gestanden haben, und dies können an der Stelle der Fensterlaibung nur Moses und Aaron gewesen sein; tatsächlich tritt Moses in San Nicola in Carcere in einer vergleichbaren Medaillonreihe auf. ${ }^{62}$ Die drei anschließenden Medaillons müssten Vertretern der beiden Bücher Josue und Richter gehört haben, also sicherlich Josue selbst sowie vielleicht Gideon und Samson.

Das erhaltene Medaillon Samuels steht zweifellos für das erste der beiden unter seinem Namen laufenden Bücher, das die Vulgata als erstes Buch der Könige auffasst, doch zugleich wohl auch für das kleine Buch Ruth, das Samuel zugeschrieben wurde. Danach ist für das zweite Buch Samuel, das zweite Buch der Könige nach der Vulgata, wohl Nathan anzunehmen, der eng mit den Schicksalen Davids verbunden ist, der in diesem Buch im Mittelpunkt steht. Für die zwei folgenden Bücher, das dritte und vierte der Könige nach der Einteilung der Vulgata, das erste und zweite Buch der Könige nach der hebräischen Bibel, sind die beiden nichtschriftlichen oder ,früheren Propheten', wie das Judentum sie nennt, Elias und Eliseus, zu vermuten, die in diesen Büchern einen wesentlicheren Teil des Geschehens bestimmen als die gleichzeitigen Könige. Auf die nichtschriftlichen Propheten scheinen, wie das aufgrund der Angaben von Eclissi gesicherte Medaillon des Königs Ezechias (716-687) anzeigt, stellvertretend für die beiden Bücher der Chronik, der Paralipomena der Vulgata, die positiven, Jahwetreuen Reformkönige Judas gefolgt zu sein. Auf Ezechias folgte der ihm in der Jahwetreue gleichende König Josias (640-609), dessen Namensrest -AS Eclissi und der Barbarini-Zeichner noch vorfanden. Dass auf diese Weise allein das

61 Symmetrie und vorgegebener Raum verlangen 12, Eclissi aber gibt auf dem Plan, auf dem er die Abschnitte für die Medaillons einzeichnet, nur 11 Namen (die elf Abschnitten entsprechen) an. Doch überspringt er in seinem Plan ABACHUK, dessen Medaillon er gleichwohl auf fol.21 groß abbildet. Auf der Südseite nennt Eclissi nur SAMUEL UND EZECHIAS sowie [Josi]AS. SAMUEL, dessen Medaillon noch erhalten ist, erscheint - da die Tondi annähernd gleich groß anzunehmen sind - im siebenten Abschnitt der Südwand, EZECHIAS im elften, [Josi]AS im zwölften Abschnitt, so verbleiben zwischen Jakob und Samuel fünf Tondo-Plätze und zwischen Samuel und Ezechias drei.

62 Vgl. dazu unten. 
Königtum Juda berücksichtigt wird, ist zweifellos darin begründet, dass Jesus nach der neutestamentlichen Auffassung in der Generatio Christi bei Matthäus und Lukas, die im Schriftband von Jacob ja implizit angesprochen wird, der davidischen Familie angehört, die aber eben nur im Südreich an der Herrschaft blieb.

Das auf dem anschließenden südlichen Abschnitt der Ostwand vorauszusetzende, doch durch den Umbau von 1570 zerstörte Medaillon wird dementsprechend die Verfasser der Bücher Esdra (bzw. Esra) oder Nehemia geboten haben, beide die politischen Führer der nachexilischen Zeit. Damit endet die Medaillonfolge der Südhälfte der Kirche, um unter Auslassung der kurzen Bücher Tobias, Judith, Esther und Iob auf der nördlichen Hälfte der Westwand erneut einzusetzen. Möglich ist allerdings, dass die Reihe der Medaillons in den im 16. Jahrhundert zerstörten Chorraum einbog, und so dort die heute fehlenden Verfasser und Bücher der Bibel Berücksichtigung fanden.

Auf dem Nordteil der Westwand treten nun - in bezeichnender Gegenüberstellung zu den ersten beiden Patriarchen Israels auf dem Südteil derselben Wand - die königlichen Stifter des Herrschersitzes Jerusalem und des Tempels mit ihren Texten auf: DAVID und SALOMON, der eine für das Psalmenbuch, dessen Verfasser er gewesen sein soll, der andere für die anschließenden vier Bücher, die sämtlich Salomon zugeschrieben wurden: das der Sprüche und des Predigers, das Hohe Lied und das Buch der Weisheit. Nun folgt auf der Nordwand die Reihe der Häupter der ,Späteren Propheten', streng in der Reihenfolge, in der ihre Schriften in der Vulgata aufeinander folgen. Nach den vier Großen Propheten erscheint eine Auswahl der Kleinen Propheten - insgesamt acht: sieben auf der Nordwand und einer, wie sich zeigte, wohl AGGEUS, auf dem Nordteil der Ostwand. Auch hier könnte die Reihe der Medaillons im Chorraum ihre Fortsetzung gefunden haben, in diesem Falle wären dann noch die letzten beiden Kleinen Propheten nach Aggeus gefolgt und für die letzten beiden Bücher der Vulgata einer der Makkabäer-Brüder, Judas oder Jonathan.

Der obere Bereich der Apsis dürfte in Entsprechung zur Westwand mit dem apokalyptischen Gehalt ein gleichwertiges christologisches Motiv geboten haben. $\mathrm{Zu}$ denken wäre in erster Linie an eine Traditio Legis, ein in Rom geläufiges Motiv. ${ }^{63}$ Da darin nach Christus auch Petrus und Paulus gezeigt werden, hätte sich so eine ,Antwort' zum Auftreten der Apostel im Vollbild des Traums von Konstantin auf der gegenüberliegenden Westwand ergeben, zugleich wäre über den in der Traditio bevollmächtigten Petrus der Anschluss an seine Nachfolger, die Inhaber des römischen Stuhls geboten gewesen.

In den ihnen zugehörigen Verfassern oder Hauptfiguren und ihren Aussagen umgaben so die Bücher des Alten Testaments den Raum. Dabei bieten die Dargestellten, wie aus den von Eclissi überlieferten oder rekonstruierbaren Schriftrollentexten

63 Monumentale Beispiele bieten in Rom: Alt St. Peter, S. Paolo fuori le mure, Sta. Constanza, S. Sebastiano al Palatino, schliesslich S.Silvestro in Tivoli; dazu und zu den antiken Werken siehe Couzin 2015. 
hervorgeht, durchweg Aussagen zum Verhältnis des weltlichen Herrschers zu Gott, zur Abhängigkeit der weltlichen Herrschaft von der überragenden Macht Gottes.

In den ihnen zugehörigen Verfassern oder Hauptfiguren und ihren Aussagen umgaben damit, wie die Benennungen der Dargestellten und die Texte der Schriftbänder zeigen, die Bücher des Alten Testaments den Raum. Dabei bieten die Dargestellten, wie aus den von Eclissi überlieferten oder rekonstruierbaren Rotulustexten hervorgeht, durchweg Aussagen zum Verhältnis des weltlichen Herrschers zu Gott, zur Abhängigkeit der weltlichen Herrschaft von der überragenden Macht Gottes. Die Bildanlage der Silvesterkapelle von Quattro Coronati ist einzigartig - und gerade dies zeigt eine Bewusstheit der Inszenierung und eine besondere Aussageabsicht an. Doch sie hat Vorläufer - und zwar eine Medaillonfolge aus San Nicola in Carcere, die im 19. Jahrhundert abgenommen wurden und als bloße Tondi in der Pinacoteca Vaticana aufbewahrt werden. Vier Medaillons alttestamentlicher Personen: MOYSES, AMOS, HYEREMIAS und AGGEUS, deren Schriftbänder sämtlich das Geschehen eines selbst textlosen größeren Tondos erläutern, der seinerseits die Epiphanie Christi bei der Taufe im Jordan erläutert.

\section{Die Bilder und Texte der Silvesterkapelle als pro- pagandistisches Aussageganzes}

Angesichts der Befunde in San Nicola müssen die Folge der Patriarchen- und Prophetentondi der Silvesterkapelle und die Aussagen ihrer Rotuli in einem konstitutivem Bedeutungszusammenhang mit den (nur noch bruchstückhaft benannten ${ }^{64}$ ) Konstantin-Silvester-Szenen der mittleren Bildzone gesehen werden, vor allem mit den beiden Bildern, die vom Text der sogenannten Konstantinischen Schenkung ausgehen und damit über die Silvesterlegende hinausgehen. Tatsächlich greifen alle gesicherten Rotulustexte der in der Kapelle auftretenden alttestamentlichen Personen inhaltlich ineinander, formulieren eine gemeinsame Aussage, die auf die Feststellung der Herrschaft Gottes und seines Erwählten zielt. Doch wenn die Inschriften auf den Rotuli von Christi Macht sprechen, so tun sie das in einem funktionalen Zusammenhang: Im Verbund mit den szenischen Bildern diskutieren die Texte der Schriftbänder die Frage der Herrschaft und zwar wesentlich hinsichtlich dessen, dass die geistliche Macht der weltlichen Macht übergeordnet sei. Dafür lassen die Planer der Malerei sämtliche Bücher der heiligen Schrift in ihren Hauptfiguren mit ihren Aussagen zu Christus auftreten, um so das eigene Dafürhalten, die Ablehnung und Verdammung einer

64 Eclissi notiert an den beiden Vollbildern die (auch an der Wand noch in Resten erkennbaren) Benennungen: unter dem Bild der ,Konstantinischen Fälschung: S. SILVESTER . CONSTANTINUS; unter der ,Stierszene‘: ... TRS ... UCUS [IU]DICES; unter der ,Kreuzaufffindung‘: [IN]VENTIO STE CRUCIS. 
nicht-geistlichen Herrschaft, als in den Aussagen der Schrift begründet zu belegen. Der Schrift aber, so ist dabei vorausgesetzt, kann nicht widersprochen werden.

Dieser impliziten Polemik entsprechend geben die in den Rotuli enthaltenen Herrschaftszuweisungen, in ihren vielen Hinweisen auf Gericht und Strafe in den Rotuli von SALOMON, YSAIAS, IEREMIAS, MICHEAS, AMOS, DANIEL JOSIAS, ABACHUC einen Kampfcharakter zu erkennen. Innerhalb des gemeinsamen Tenors setzen dann einige Schriftbänder einen besonderen Akzent. Ihre christologischen Aussagen lassen inhaltliche Bezüge erkennbar werden, die als Feststellungen einer überragenden Verfügungsgewalt des Nachfolgers des von Christus beauftragten Petrus zu verstehen und auf konkrete Ereignisse zu beziehen sind. Das Wort Jacobs: NON AUFERETUR SCEPTRUM / DE JUDA ET DUX DE F(EMORE EIUS) enthält den Anspruch auf das Szepter und den kriegerisch-weltlichen Dukat, der den Kern des Imperatorentum ausmacht, und dieser Anspruch eignet - so folgt aus der Verbindung der Tondi mit den Szenen der Constitutum-Fälschung - dem Nachfolger Petri, dem römischen Patriarchen: Als ,Stellvertreter Christi“ wird er als Juda-Nachkomme gesehen, ihm kommen daher Szepter und Dukat zu - bis zur Wiederkunft Christi (wie der Folgevers sagt). Eben daher sind die Stammväter des Volkes Gottes so ausdrücklich als ,Patriarchen' wiedergegeben und ihre Kopfbedeckung dem phrygium Silvesters angeglichen: Sie sind als Träger der angekündigten, in Gott ruhenden Herrschaft Vorläufer des römischen Patriarchen. Was Konstantin Silvester zuerkannte, wird damit als schon uranfänglich der Verfügung des Kaisers entzogen dargestellt. Und dies festigt dann der weitere Sinn des David-Wortes, wenn er Gott verkünden lässt, dass er Davids Nachkommenschaft auf den eigenen Thronsitz (super sedem meam) setzen werde. Das meint zwar zunächst Christus, strahlt aber doch zugleich auf den aus, der sich als dessen Stellvertreter im Irdischen zu inszenieren sucht.

Die aus den Buchstabenresten rekonstruierten Schriftbänder fächern das Aussagenspektrum der von Eclissi voll wiedergegebenen Schriftbänder im selben Sinne auf. Besonders im Vers des MICHEAS wird dies deutlich: DE SION EGREDIETUR LEX / ET VERBUM DOMINI DE HIERUSALEM. Denn ,Sion“ war nicht nur der Hauptort von Jesu Erlösungswirken, sondern auch ein seit langem eingeführter Titel der Kirche, als deren Oberhaupt der römische Patriarch sich sah. Von ihr, von ihm also ginge demnach das ,Gesetz‘ aus. In Verbindung mit dem Thronen Silvesters und dem Stratordienst des Kaisers bringt die Äußerung DE SION EGREDIETUR LEX den Anspruch des Papstes auf verbindliche Rechtsetzung zum Ausdruck - und dies unter Einschluss des zum thronenden Papst herantretenden Kaisers. Der zweite Teil des Verses, eigentlich ja nur ein Parallelismus zum Vorhergehenden, kann dann noch eine weitere Aussagekomponente aussprechen und nach der Rechtsetzung die Rechtsprechung (die ja auch der Rotulus von SALOMON hervorhebt) thematisieren. Vom Haupt der römischen Kirche geht das Wort, der Spruch des Herrn aus, der als solcher die Verfügbarkeiten eines Königs oder Kaisers übersteigt. Dies muss - so die Sicht der Planer der Silvesterkapelle - allen Zeitgenossen als unangreifbare Rechtfertigung der vom Papst ausgesandten Bannsprüche gelten. 
Vor diesem Hintergrund tönt auch das Wort des AMOS mit den drei darin auftretenden ,Personen“ auf besondere Weise: Der erscheinende Herr fordert den angesprochenen offensichtlich spirituellen ,Seher' auf, im Zuge eines Strafgerichts den Türsturz und damit den ganzen Bau zu zerschlagen, und versichert, dass den so Gestraften kein Ausweg bleibe. In den Jahren um 1245 musste das den ,Bau' des Imperium meinen. So wird das zweideutige ,Reis ‘ (virga) des ISAIAS-Wortes in seiner strafenden Bedeutung aufgenommen und verstärkt. Das DANIEL-Zitat Jesu (ähnlich die erste Zeile des JOSIAS-Rotulus) führt dies dann weiter, redet von Auslieferung und Verwüstung des heiligen Ortes, was zeitgeschichtlich nahezu zwangsläufig als Hinweis auf Rom klingen musste, vor das Kaiser Friedrich II. drohend gezogen war, und fordert die erkennenden Gottverbundenen zur Flucht aus der Stadt auf. Dies kann in der gegebenen Sprechsituation nur die Führer der Kirche meinen und insbesondere den Papst, der eben Rom verlassen hatte, um Gottes Wort ,getreu' die Herrschaft des ,gottlosen' Kaisers mit dem Bann zu zerschlagen. Der unmittelbare Vollzug des Allgemein-Richterlichen fehlt der Darstellung des endzeitlichen Christus auf der Westwand also gerade deswegen, weil ein besonderes ,richterliches Auftreten' ausgedrückt werden soll, weil der nun sich als Stellvertreter Christi auf Erden inszenierende Papst das in den Beischriften der Rotuli angekündigte ,Gerichtliche“ auf sein eigenes Tun bezogen sehen will: auf seinen Bannspruch gegen den Einen, Friedrich II., den Kaiser.

\section{Text und Bild im Raum}

Vor diesem Hintergrund erst wird das Ganze der textualen und piktoralen Rauminszenierung der Silvesterkapelle verständlich. Die heute großteils zerstörten, doch durch die Dokumentation des 17. Jahrhunderts überlieferten oder - wie hier vorgestellt erschließbaren Texte, sowohl die Benennungen der Repräsentanten der biblischen Bücher wie die Aufschriften ihrer Rotuli, sind das entscheidende Moment - und dies gerade durch ihre Ordnung im Raum. Die auftretenden Sprecher und ihre Aussage umringen mit ihren Rückverweisen auf das alles begründende verbindliche Schriftwerk jeglichen Vollzug in diesem - im Falle der Eroberung des Lateran letztverfügbaren - päpstlichen Herrschaftsraum. Sie ,bedeuten', erläutern und untermauern die Bildaussagen der aus vier verschiedenen Texttraditionen abgeleiteten szenischen Bilder, perspektivieren das in ihnen Gezeigte und schärfen die Bilder inhaltlich, machen das in ihnen Gezeigte und Verlangte gleichsam unwiderlegbar. Mit den Versicherungen der eigenen Identität in den Texten, mit den darin enthaltenen richterlichen Ansprüchen und Drohungen wird das Selbstbild der Kurie und des römischen Patriarchen umfassend begründet. Das umfasst alle Bilder, nicht nur die bekannte ,Tiara-Übergabe“ und den ,Strator-Dienst‘, sondern gerade auch die Bilder der Südseite: das Bild von Silvesters Disput mit Zambri, das die Verfügung des Papstes über das bannende, ja tötende Wort kennzeichnet, das Bild der ,Kreuzauffindung‘, durch 
das die entscheidende, wirkmächtigste Reliquie in die Hand des römischen Bischofs gelangte und zuletzt - unverhohlen auf den Kaiser weisend - das Bild der ,Drachenfesselung', das beispielhaft die Macht dieses Mittels in der päpstlichen Hand vergegenwärtigt. Sie alle veranschaulichen, dass das - nach papalistischer Deutung - in den Texten Verkündete nicht vergangen, sondern über Petrus und Silvester unverrückbar in der Macht von deren Nachfolgern liegt. So ist in diesem unauflösbaren Ineinander von Text und Bild in dem ,lateranischen' päpstlichen Herrschaftsbau von S. Silvestro das übersteigerte Selbstbild des Papsttums in der Zeit Friedrichs II. Ausdruck geworden. Jedes Tun und Meinen hatte sich so vor den - gleichsam als geöffnetes Altes Testament - den Raum umfassenden Worten der von Gott Ergriffenen zu bewähren, ihre Gebote zu beachten.

\section{Literaturverzeichnis}

Barberini, Giulia (1993), The Basilica of the Santi Quattro coronati in Rome, Rom.

Berschin, Walter (1980), „Drei griechische Maiestas-Tituli in der Trier-Echternacher Buchmalerei“, in: Frühmittelalterliche Studien 14, 299-309.

Concina, Ennio (1996), Kirchen in Venedig: Kunst und Geschichte. Photographien von Piero Codato und Vittorio Pavan, München (Originalausgabe Udine 1995).

Couzin, Robert (2015), The Traditio Legis: Anatomy of an Image, Oxford.

De Marchi, Andrea G. (1993): „Eclissi, Antonio“, in: Dizionario Biografico degli italiani $42<\mathrm{http}$ :// www.treccani.it/enciclopedia/antonio-eclissi_\%28Dizionario-Biografico\%29/> (Stand Januar 2018).

Draghi, Andreina (2006), Gli affreschi dell'Aula gotica del Monastero dei Santi Quattro Coronati: una storia ritrovata, Mailand.

Eichmann, Eduard (1930), „Das officium stratoris et strepae“, in: Historische Zeitschrift 124, 16-40.

Forcella, Vincenzo (1876), Iscrizioni delle chiese e di alteri edifici di Roma dal secolo X fino ai giorni nostri, Bd. 8, Roma.

Fried, Johannes (2007), Donation of Constantine and Constitutum Constantini. The Misinterpretation of a Fiction and its original Meaning, Berlin / New York 2007.

Fried, Johannes (2011), „Die Konstantinische Schenkung“, in: Johannes Fried u. Olaf B. Rader (Hgg.): Die Welt des Mittelalters. Erinnerungsorte eines Jahrtausends, München, 295-311.

Fuhrmann, Horst (Hg.) (1968), Das Constitutum Constantini (Monumenta Germaniae Historica: Fontes iuris germanici antiqui in usum scholarum ex monumentis Germaniae historicis separatim editi 10), Hannover.

Ghetti, Bruno Maria Apollonij (1964), I Santi Quattro Coronati, Roma.

Herklotz, Ingo (2006), „Vätertexte, Bilder und lebendige Vergangenheit. Methodenproblem in der Liturgiegeschichte des 17. Jahrhunderts“, in: Bernd Carqué, Daniela Mondini u. Matthias Noell (Hgg.): Visualisierung und Imagination: Materielle Relikte des Mittelalters in bildlichen Darstellungen der Neuzeit und Moderne, Bd.1, Göttingen, 215-252.

Holtzmann, Robert (1928), Der Kaiser als Marschall des Papstes. Eine Untersuchung zur Geschichte der Beziehungen zwischen Kaiser und Papst im Mittelalter, Berlin / Leipzig.

Holtzmann, Robert (1932), „Zum Strator und Marschalldienst. Zugleich eine Erwiderung“, in: Historische Zeitschrift 145, 301-350. 
Kantorowicz, Ernst H. (1961), „Constantinus Strator. Marginalien zum Constitutum Constantini“, in: Alfred Stuiber u. Alfred Hermann (Hgg.), Mullus. Festschrift für Theodor Klauser (Jahrbuch für Antike und Christentum, Ergänzungsband 1), Münster, 181-189.

Krautheimer, Richard (1970), „SS. Quattro Coronati“, in: Corpus Basilicarum Christianarum Romae Vol. IV, Città del Vaticano.

Manzi, Pietro (1968), Il convento fortificato dei SS. Quattro Coronati nella storia e nell' arte, Roma. Mazal, Otto/Unterkircher, Franz (Hgg.) (1967), Katalog der abendländischen Handschriften der Österreichischen Nationalbibliothek. ,Series Nova“ (Neuerwerbungen), Bd. 3, Wien.

Mazal, Otto (1981), Byzanz und das Abendland (Katalog), Wien 1981.

Matthiae, Guglielmo (1964), Pittura politica del Medioevo romano, Roma.

Matthiae, Guglielmo (1966), Pittura Romana del Medioevo II, Roma.

Mitchell, John (1980), „St.Silvester and Constantine at the SS.Quattro Coronati“, in: Federico I/ $e$ l'Arte del duecento Italiano (Atti della III. Settimana di studi di storia dell'Arte medievale dell' università di Roma, 15-20 maggio 1978), Bd. 2, Rom, 15-32.

Mombritius, Boninus (1910), Sanctuarium seu Vitae Sanctorum. Novam hanc editionem curaverunt duo monachi Solesmenses, Bd. 2, Paris.

Mommsen, Theodor (Hg.) (1904), Codex Theodosianus, Bd.1, Berlin.

Muñoz, Antonio (1913), „Affresci del XII secolo ai Ss. Quattro Coronati“, in: Studi Romani 1, Roma, 278-279.

Muñoz, Antonio (1914), Il restauro della Chiesa e del Chiostro dei SS. Quattro Coronati, Roma.

Noll, Thomas (2011), Die Sylvesterkapelle in SS. Coronati in Rom, Berlin.

Pohlkamp, Wilhelm (1983), „Tradition und Topographie: Papst Silvester I. (314-335) und der Drache vom Forum Romanum“ (Diss. Münster, 1982), in: Römische Quartalschrift für Christliche Altertumskunde und Kirchengeschichte 78, 1-100.

Schneider, Wolfgang Christian (1991), „Die Generatio Imperatoris in der Generatio Christi. Ein Motiv der Herrschaftstheologie Ottos III. in Trierer, Kölner und Echternacher Handschriften“, in: Frühmittelalterliche Studien 25, 226-258.

Schramm, Percy Ernst (1983), Die deutschen Kaiser und Könige in Bildern ihrer Zeit: 751-1190. Neuauflage, München.

Seroux d'Agincourt, Jean Baptiste L. G. (1825), Storia dell'Arte col mezzo dei Monumenti dalla sua decadenza nel IV secolo fino al suo risorgimento nel XVI, Bd. 6, Milano.

Sohn, Andreas (1997), „Bilder als Zeichen der Herrschaft. Die Silvesterkapelle in SS. Quattro Coronati (Rom)“, in: Archivum Historiae Pontificae 35, 7-47.

Walter, Christopher (1970), „Papal Political Imagery in the Medieval Lateran Palace“, in: Caltiers archéologiques 20, 157-160, 170-176.

Winkelmann Eduard (Hg.) (1964), Acta imperii inedita saec. XIII et XIV, Bd. 2 (Innsbruck 1885), Nachdruck, Aalen.

Zahlten, Johannes (1994): „Barocke Freskenkopien aus SS.Quattro Coronati in Rom: Der Zyklus der Silvesterkapelle und eine verlorene Kreuzigungsdarstellung“, in: Römisches Jahrbuch der Bibliotheca Hertziana 29, 19-43.

\section{Photo Credits}

Fig. 1-8: Wolfgang Christian Schneider.

Fig. 9-16: Aquarelle von Antonio Eclissi, Codex Series nova 3311, Österreichische Nationalbibliothek, Wien. 